\title{
Safety and feasibility of the NanoKnife system for irreversible electroporation ablative treatment of canine spontaneous intracranial gliomas
}

\author{
*John H. RossmeisI Jr., DVM, ${ }^{1-3}$ Paulo A. Garcia, PhD,, ${ }^{2,3}$ Theresa E. Pancotto, DVM, ${ }^{1}$ \\ John L. Robertson, VMD, PhD, ${ }^{1-3}$ Natalia Henao-Guerrero, DVM, ${ }^{1}$ Robert E. Neal II, PhD, ${ }^{3}$ \\ Thomas L. Ellis, MD, ${ }^{4}$ and Rafael V. Davalos, $\mathrm{PhD}^{3}$ \\ 1Department of Small Animal Clinical Sciences and ${ }^{2}$ Veterinary and Comparative Neuro-oncology Laboratory, Virginia-Maryland \\ Regional College of Veterinary Medicine, and ${ }^{2}$ Virginia Tech-Wake Forest University School of Biomedical Engineering and \\ Sciences, Virginia Tech, Blacksburg, Virginia; and ${ }^{4}$ Department of Neurosurgery and Deep Brain Stimulation Program, School of \\ Medicine, Wake Forest University, Winston-Salem, North Carolina
}

\begin{abstract}
OBJECT Irreversible electroporation (IRE) is a novel nonthermal ablation technique that has been used for the treatment of solid cancers. However, it has not been evaluated for use in brain tumors. Here, the authors report on the safety and feasibility of using the NanoKnife IRE system for the treatment of spontaneous intracranial gliomas in dogs.

METHODS Client-owned dogs with a telencephalic glioma shown on MRI were eligible. Dog-specific treatment plans were generated by using MRI-based tissue segmentation, volumetric meshing, and finite element modeling. After biopsy confirmation of glioma, IRE treatment was delivered stereotactically with the NanoKnife system using pulse parameters and electrode configurations derived from therapeutic plans. The primary end point was an evaluation of safety over the 14 days immediately after treatment. Follow-up was continued for 12 months or until death with serial physical, neurological, laboratory, and MRI examinations.
\end{abstract}

RESULTS Seven dogs with glioma were treated. The mean age of the dogs was $9.3 \pm 1.6$ years, and the mean pretreatment tumor volume was $1.9 \pm 1.4 \mathrm{~cm}^{3}$. The median preoperative Karnofsky Performance Scale score was 70 (range 30-75). Severe posttreatment toxicity was observed in 2 of the 7 dogs; one developed fatal (Grade 5) aspiration pneumonia, and the other developed treatment-associated cerebral edema, which resulted in transient neurological deterioration. Results of posttreatment diagnostic imaging, tumor biopsies, and neurological examinations indicated that tumor ablation was achieved without significant direct neurotoxicity in 6 of the 7 dogs. The median 14-day post-IRE Karnofsky Performance Scale score of the 6 dogs that survived to discharge was 80 (range 60-90), and this score was improved over the pretreatment value in every case. Objective tumor responses were seen in $4(80 \%)$ of 5 dogs with quantifiable target lesions. The median survival was 119 days (range 1 to $>940$ days).

CONCLUSION With the incorporation of additional therapeutic planning procedures, the NanoKnife system is a novel technology capable of controlled IRE ablation of telencephalic gliomas.

http://thejns.org/doi/abs/10.3171/2014.12.JNS141768

KEY WORDS brain tumor; dog; electroporation; glioma; neurosurgery; oncology

$\mathrm{F}$ OR the past several decades, the prognosis for patients with a malignant glioma has remained relatively static and poor. ${ }^{48}$ During this time, many novel therapies that have shown preclinical promise for the treatment of malignant gliomas have been developed, but few have been translated successfully to the clinic. Despite the progress being made with transgenic and genetically engineered rodent glioma models, a significant

\footnotetext{
ABBREVIATIONS CTCAE = Common Terminology Criteria for Adverse Events; DICOM = Digital Imaging and Communications in Medicine; GBM = glioblastoma multiforme; IRE = irreversible electroporation; IV = intravenous; KPS = Karnofsky Performance Scale; PBS = phosphate-buffered saline; PD = progressive disease; PDMS = polydimethylsiloxane; $\mathrm{PGP}=$ probe guide pedestal; RANO = response assessment in neurooncology; $\mathrm{SD}=$ stable disease. SUBMITTED July 31, 2014. ACCEPTED December 12, 2014. INCLUDE WHEN CITING Published online July 3, 2015; DOI: 10.3171/2014.12.JNS141768.

DISCLOSURE The authors report no conflict of interest concerning the materials or methods used in this study or the findings specified in this paper. AngioDynamics, Inc. loaned the NanoKnife system for and manufactured the electrodes used in the study. The following grants funded this study: the Wallace $H$. Coulter Foundation Early Career Translational Research Awards and National Science Foundation CBET-0933335 and CAREER CBET-1055913. Drs. Rossmeisl, Garcia, Robertson, Neal, and Davalos hold patents and have patents pending on irreversible electroporation technology for tissue ablation.

* Drs. Rossmeisl and Garcia contributed equally to this work.
} 
obstacle to therapeutic development remains the failure of animal model systems to faithfully reproduce phenotypically and genotypically diverse tumors on a scale comparable to that seen in humans. ${ }^{6}$

Dogs with spontaneous brain tumors have been recognized for their potential as a large translational animal model for preclinical assessment of novel therapeutics because significant clinical, cytogenetic, epidemiological, and pathophysiological similarities have been identified between canine and human brain tumors. ${ }^{6,23}$ Although the true incidence of canine brain tumors is unknown, necropsy-based estimates indicate a frequency of $2 \%$ for primary tumors, which approximates the autopsy frequency of CNS tumors seen in humans. ${ }^{30}$ Currently, there are limited data regarding the clinical efficacy of conventional therapies in dogs with a histopathologically confirmed glioma, but existing evidence suggests that the prognosis is poor, irrespective of the type of treatment administered. ${ }^{20,39}$ The homologies between the diagnostic imaging and histopathologic features of canine and human gliomas have been well described. ${ }^{27,39,43,49}$ The molecular characterization of canine and human gliomas has revealed several common denominators, including the presence of cancer stem-like cells and the expression profiles of growth factors and other molecular biomarkers such as epidermal growth factor, platelet-derived growth factor, interleukin $13 \mathrm{R} \alpha 2$, telomerase activity, and vascular endothelial growth factor., ${ }^{9,11,36,43,44}$ Although the genetic characterization of canine gliomas is in its infancy, the field has advanced rapidly since the publication of the canine genome. ${ }^{45}$ In addition, the identification of genetic abnormalities in canine gliomas can be facilitated by the relative genetic homogeneity that exists in the brachycephalic dog breeds in which gliomas are overrepresented..$^{36,43}$ Thus, dogs are an attractive preclinical model, because the size and heterogeneity of canine gliomas more faithfully represent those of humans.

Pulsed electrical fields have been investigated for the treatment of malignant glioma, mainly for their cytostatic potential, as with tumor-treating fields. ${ }^{24}$ In addition, other therapies aim to facilitate local tumor treatment by enhancing chemotherapeutic drug delivery through the reversible permeabilization of the blood-brain barrier and tumor cell membranes in a process known as electrochemotherapy. ${ }^{1,40}$ Irreversible electroporation (IRE) is a novel, minimally invasive, nonthermal tissue-ablation technique that involves the placement of small electrodes into a targeted area and delivery of carefully selected electrical pulses. ${ }^{8}$ When the applied electrical field exceeds critical tissue-specific thresholds, cells within the treatment region are permeabilized irreversibly through the formation of nanoscale aqueous pores in the cytoplasmic membranes, which results in cellular death. ${ }^{8}$ In animal models with experimentally induced and naturally occurring cancers, IRE has been shown to be a safe and efficacious method of tumor ablation. ${ }^{2,34}$ The NanoKnife system (AngioDynamics, Inc.) has been used safely for ablating a variety of softtissue malignancies in humans, including those in the pancreas, liver, lung, prostate, and kidney.,5,29,31,46 In addition to its minimally invasive nature and nonthermal mode of action, IRE offers other advantages in the form of rapidity of treatment, which is on the order of minutes. ${ }^{13}$ IRE therapy also has the potential to spare the vasculature, ductal networks, and extracellular matrix, which facilitates posttreatment healing. $2,8,35$

Motivated by evidence of the positive effect that the extent of resection confers on the survival of human patients with high-grade astrocytoma treated with radiotherapy and the technical challenges that often preclude grosstotal glioma resection, our laboratory has focused on leveraging the advantages associated with IRE for use in the ablative treatment of malignant glioma.$^{50}$ Using canine and rodent models, we have demonstrated that IRE can be used to ablate normal and neoplastic brain tissue safely and predictably. ${ }^{13,16-18}$ We and others have also shown that a transient and reversible disruption of the blood-brain barrier occurs in a penumbra of tissue surrounding the zone of IRE ablation, which can be exploited for the delivery of high-molecular-weight chemotherapeutics to microscopic tumor infiltrates that extend beyond the gross-tumor margins, which account for the majority of local treatment failures in patients with malignant glioma. ${ }^{13,17,19,21}$

We hypothesized that these features of IRE coupled with advances in therapeutic planning would allow for the safe and precise ablation of intracranial gliomas. The specific aim of this study was to evaluate the safety of IRE performed with the NanoKnife system for the treatment of spontaneous glioma in dogs.

\section{Methods \\ Study Design}

This was a prospective single-center study conducted to evaluate the safety and feasibility of the techniques necessary for ablative treatment of spontaneous canine intracranial glioma using the NanoKnife system. Study dogs were treated according to the standards established by the Guide for Care and Use of Laboratory Animals, and all study procedures were approved by the institutional animal care and use committee and the Virginia-Maryland Regional College of Veterinary Medicine Veterinary Teaching Hospital clinical review board.

Client-owned dogs with a suspected spontaneous telencephalic glioma based on MRI characteristics were candidates for the study. For inclusion, each dog was required to have a diagnostic MRI within 2 weeks of enrollment that demonstrated a single telencephalic intraaxial mass lesion with imaging features consistent with those of glioma, ${ }^{49}$ have stable cardiopulmonary and neurological functions, and have no evidence of concurrent systemic malignancy or significant hepatic or renal dysfunction. Exclusion criteria included previous treatment of the brain lesion with any type of radiotherapy. The dogs were enrolled using a passive approach after identification from the routine clinical population presented to the study center or its referral affiliates; recruitment occurred after an investigator's review of clinical data from each candidate. Each client provided written informed consent for his or her dog to receive the protocol treatment. Those dogs in which the NanoKnife electrode probes were inserted into the brain comprised the intent-to-treat population.

The primary study end point was to evaluate the safety 
of IRE with the NanoKnife system for the treatment of intracranial glioma. Safety was defined as the absence of severe clinical toxicity within 14 days of the IRE procedure. Severe clinical toxicity was defined by a $\geq 20$-point decline in the Karnofsky Performance Scale (KPS) score from the pretreatment value ${ }^{47}$ or the development of Grade 3,4 , or 5 (severe, life-threatening, or fatal, respectively) adverse events, as classified and graded according to the Cancer Therapy Evaluation Program Common Terminology Criteria for Adverse Events (CTCAE) version 3.0 (http://ctep.cancer.gov/protocolDevelopment/electronic applications/docs/ctcaev3.pdf).

Secondary end points included direct toxicity evaluations determined from posttreatment imaging studies, including induction or exacerbation of brain edema, procedure-associated hemorrhage or infarction, or collateral damage to brain tissue outside of IRE-treated regions. Additional secondary end points included changes in KPS score at 7 and 14 days of treatment, results of therapeutic response assessments using response assessment in neurooncology (RANO) criteria, ${ }^{37}$ and overall survival, as determined from the date of IRE treatment to the date of death or being killed humanely. The administration of additional therapies was permitted after the initial 14-day posttreatment observation period. Any additional therapies and their influence on outcome are reported as clinical observations only. The dogs were followed up posttreatment for a minimum of 12 months or until death or being killed humanely, whichever occurred first. Physical and neurological examinations were performed and KPS scores and adverse events noted daily during hospitalization and on Days 7, 14, 42, 90, 180, 270, and 365. Laboratory assessments were performed 1 day before IRE (Day -1 ) and on Days 1, 2, and 14. Follow-up MRI examinations of the brain were performed with the dog under general anesthesia on Days 42, 90, 180, 270, and 365 or as dictated by the dog's clinical status. The long-term assessment of the seizure outcome was performed using the Engel classification, determined from the date of discharge until death or completion of the follow-up period as follows: Class I, dogs that were completely seizure free or had convulsions only after antiepileptic drug withdrawal; Class II, dogs with rare disabling seizures, up to 3 per year; Class III, worthwhile improvement; and Class IV, no improvement. ${ }^{14}$

\section{Objective Tumor-Response Assessment}

Volumetric tumor quantification was determined by using image-analysis software (OsiriX Imaging Software version 4.1.2; OsiriX Foundation). Volumes were defined from T2-weighted MR images, because not all tumors demonstrated contrast enhancement. Manually defined regions of interest (ROIs) were generated for individual contiguous MR-image slices, and volumes were calculated with ROI-volume software.

Therapeutic responses were defined according to principles described in the RANO criteria, adapted for use in clinical veterinary medicine. ${ }^{37}$ For the purposes of this study, in dogs with quantifiable target lesions, a partial response was defined as a $\geq 50 \%$ reduction in the enhancing tumor sum products of diameters (SPD), stable disease (SD) was defined as a $<50 \%$ decrease or $<25 \%$ increase in contrast-enhancing SPD, and progressive disease (PD) was defined as a $>25 \%$ increase in contrast-enhancing SPD. For dogs with quantifiable target lesions using RANO criteria, attempts were made to perform posttreatment MRI scans and tumor biopsies under general anesthesia within 96 hours of IRE, depending on the dog's clinical status.

\section{The NanoKnife System}

The NanoKnife system includes a generator and singleuse disposable electrode probes. The NanoKnife generator is a microprocessor-controlled low-energy direct-current IRE device that operates outside of the sterile surgical field and consists of an LCD display, console and keyboard, power unit and cord, and double footswitch. The generator can deliver between 500 and $3000 \mathrm{~V}$ of energy in a maximum of 100 pulses, which have a maximum pulse length of $100 \mu \mathrm{sec}$, and it has user-controlled settings for voltage, pulse length, and number of pulses applied. The generator is designed for use in conjunction with up to 6 NanoKnife electrode probes, which work in a 2-pole operating mode in which one pole is the positive supply and the second pole is the negative return. After activation of the footswitch, low-energy direct current is transmitted from the generator to the electrode probes placed in the target area for soft-tissue ablation. The NanoKnife system monitors and visually displays the delivered voltage and current at the completion of the treatment. As a safety measure, the NanoKnife system will automatically suspend pulse delivery at any point during the treatment if a 50-A current threshold is exceeded.

For the purposes of this study, the 50- $\mu$ sec pulses used were configured with an alternating polarity between each set of 10 pulses to minimize charge build-up on the electrode surface and reduce the total charge delivered to the brain. ${ }^{13,18}$ The NanoKnife can be programmed to deliver the pulse sequence with alternating sets as long as a verification code that prohibits the automatic reorganization of the pulse order is introduced.

The electrode probes used in this study were monopolar prototypes (1-mm outer diameter) specifically modified by the manufacturer (200-104302; AngioDynamics, Inc.) with an overall length of $15 \mathrm{~cm}$ and blunted tips (Fig. $1)$, which were connected to the pulse generator via 1.8$\mathrm{m}$ insulated cables, for insertion into the brain. The active exposure length of the electrode tip can be adjusted in 5-mm increments over a range of $40 \mathrm{~mm}$. To preserve the constraints of the multielectrode probe configurations relative to each other and the dog according to our pretreatment plans, polydimethylsiloxane (PDMS) electrode immobilizers were also manufactured (Fig. 1) in our laboratory. These PDMS immobilizers readily attached to the stereotactic head frames and enabled wide latitude in the electrode probe configuration and preoperative assembly and sterilization of the electrodes.

\section{Therapeutic IRE Planning Procedure (Protocol Day -1)}

\section{Stereotactic Procedure and Dog Preparation}

The procedure is summarized in Fig. 2. On the day before IRE after clinical examinations and laboratory as- 


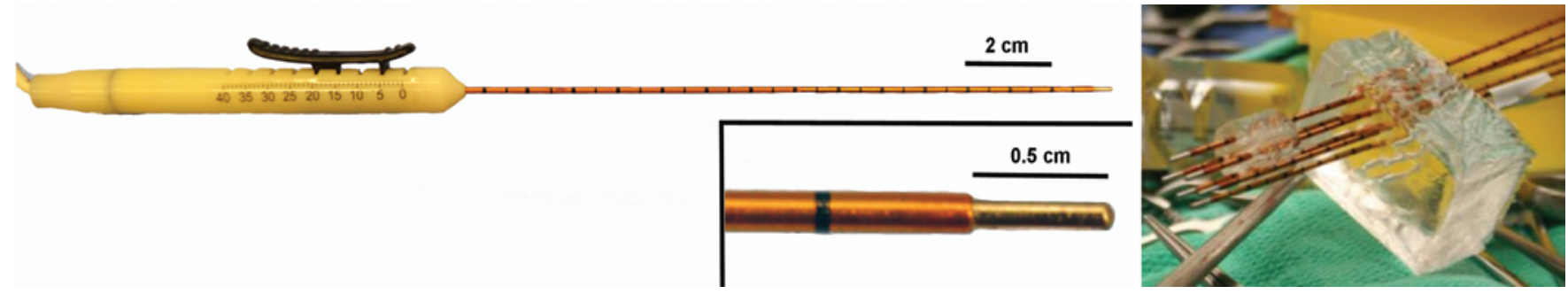

FIG. 1. Left: Blunt-tipped monopolar electrode probe used for IRE treatment of glioma. Right: PDMS immobilization device used to maintain constraints of up to a 6 -electrode probe array, as used in Cases 4 and 5 . Figure is available in color online only.

sessments, general anesthesia was induced after a 12-hour fast. The head of each dog was then affixed to a custommodified small-animal stereotactic head frame (1530 M; David Kopf Instruments) using a vinyl-polysiloxane putty (Express STD; 3M ESPE Dental Products) dental impression of the maxillary arcade. The head frame was modified by manufacturing the rostral/caudal and ear bars of the frame with polyoxymethylene acetal resin (Delrin POM; DuPont). Three-point immobilization occurred using the bite plate, ear bars, and memory foam placed under the mandible. In addition, to facilitate placement of multielectrode probe arrays (Fig. 1) into the tumors for IRE treatment, a polyoxymethylene acetal resin center-of arc quadrant apparatus that could be affixed to the rostral/ caudal bars of the head frame was also fabricated (Fig. 3).

Precontrast and postcontrast (intravenous [IV] iohexol, $0.45 \mathrm{ml} / \mathrm{kg}$ ) CT scans were obtained with a 16-slice scanner (Aquilion; Toshiba) with the dog in the fully instrumented head frame in sternal recumbency. Immediately after the CT scan, the dog was recovered from the anesthesia. Because cerebral edema induced by IRE ablation was recognized in a previous study in the normal canine brain, ${ }^{13}$ edema prophylaxis consisting of $20 \%$ mannitol (IV, $1 \mathrm{~g} / \mathrm{kg}$ every 12 hours), furosemide (IV, $0.75 \mathrm{mg} / \mathrm{kg}$ every 12 hours), and dexamethasone (IV, $0.25-0.5 \mathrm{mg} / \mathrm{kg}$ every 12 hours) was initiated 12 hours before IRE.
Day -1

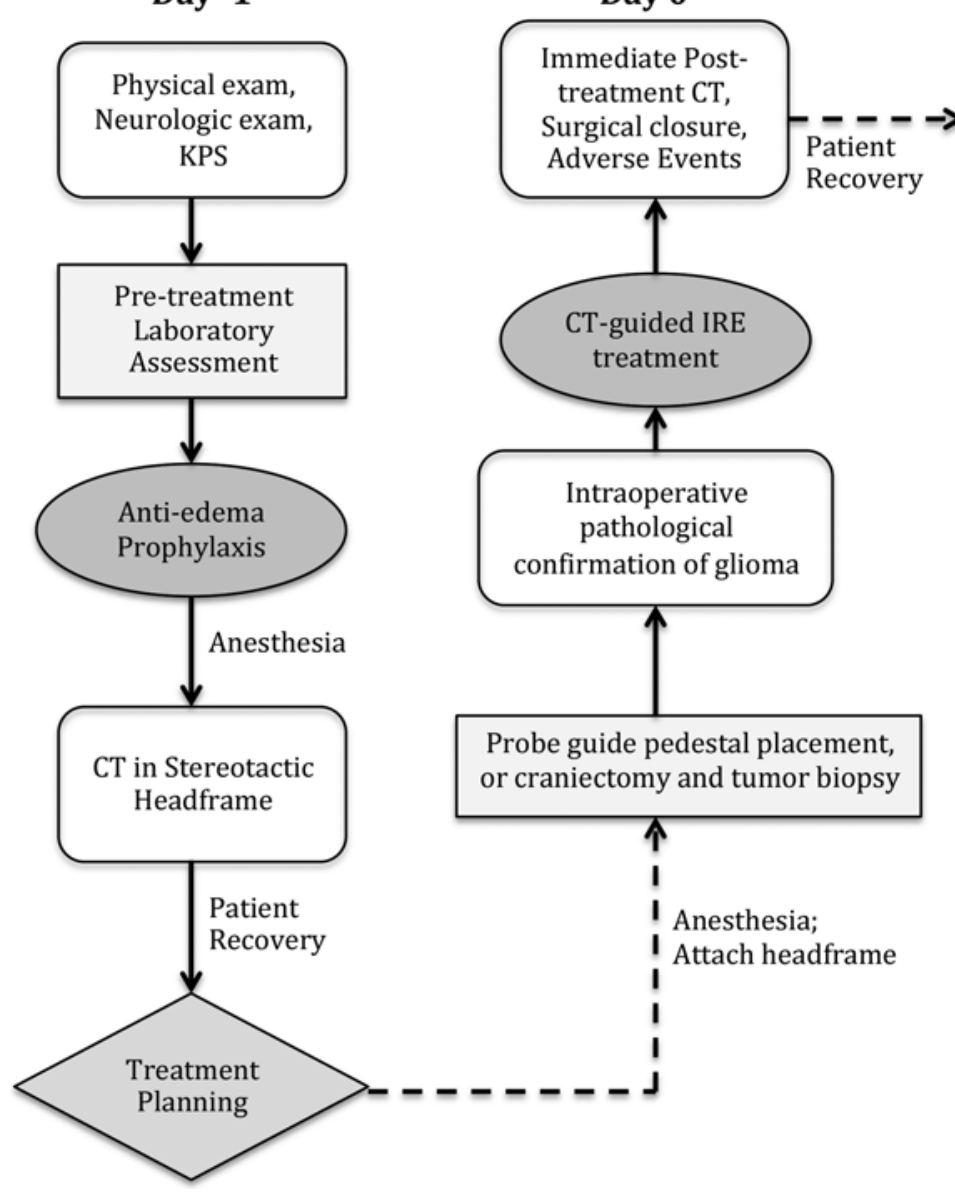

Days $+1,+2 \ldots$

Physical exam, Neurologic exam, KPS, Adverse Events

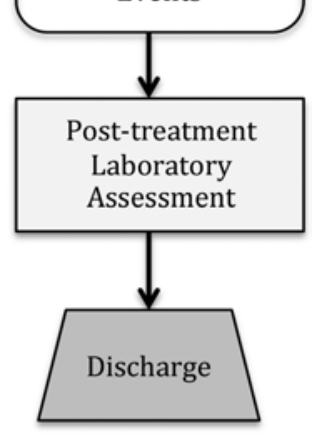

FIG. 2. Protocol for the ablation of canine intracranial gliomas with the NanoKnife system. 


\section{Stereotactic Planning}

Digital Imaging and Communications in Medicine (DICOM)-formatted images of the stereotactic CT and diagnostic MR images obtained in proximity to study enrollment were imported into image-analysis software packages (OsiriX version 4.1.2 and Mimics version 14.1 [Materialise]), and the stereotactic coordinates for tumor biopsy and electrode placement were determined. The trajectories chosen for electrode placement were generally similar to those used for biopsy. Depending on the size and geometry of the tumor, pulse delivery at multiple electrode probe depths along the same trajectory was sometimes required. The rostral/caudal coordinates were determined based on the ear-bar linear reference markers. Medial/lateral and dorsal/ventral coordinates were measured directly from DICOM-formatted images using osseous anatomical landmarks, including the external sagittal crest and external surface of the skull, respectively.

\section{Segmentation and Meshing of Brain Tissue Components}

Mimics version 14.1 image-analysis software was used to segment the tumor geometry from normal brain tissue components, including the ventricles and the white and gray matter (Fig. 4). The tumors were traced in each of the 2D diagnostic MR, CT, or any other DICOM-format imaging modalities according to intensity values. A solid $3 \mathrm{D}$ representation of the tumor volume, the ventricles, and the brain tissue was then refined and exported to 3-matic version 6.1 (Materialise) to generate a volumetric mesh that was compatible with the computational modeling software.

\section{Finite Element Modeling of Electric Field Distribution}

Refined volumetric meshes were imported into finite element modeling (Multiphysics, version 4.2a; Comsol) software to simulate the physical effects of the electric pulses in the tumor and surrounding normal brain (Fig. 4). The methods used to generate the electric field distributions in tissue are similar to the ones described by Edd and Davalos ${ }^{12}$ but include conductivity functions that reflect the nonlinear increase that occurs during electroporation in addition to white matter anisotropy. ${ }^{17}$ The electric field distribution associated with the electric pulse is given by solving the continuity equation,

$$
-\nabla \cdot(\sigma(|E|) \nabla \varphi)=0
$$

where $\sigma(|E|)$ is the electric field-dependent conductivity of the tissue and $\varphi$ is the electric potential. Boundary conditions most often include surfaces for which electric potential is specified, as in the case of a source or sink electrode, or surfaces that are electrically insulating, as on the free surfaces of the tissue, for example. The electrical boundary condition along the tissue that is in contact with the energized electrode is $\varphi=V_{0}$. The electrical boundary condition at the interface of the other electrode is set to ground. The remaining boundaries are treated as electrically insulating.

The models are fully defined and readily solvable using numerical methods once an appropriate set of boundary conditions and physical properties of the tissue are defined. The computations were performed with a commercial finite element package (Multiphysics version 4.2a). The analyzed domain extends far enough from the area
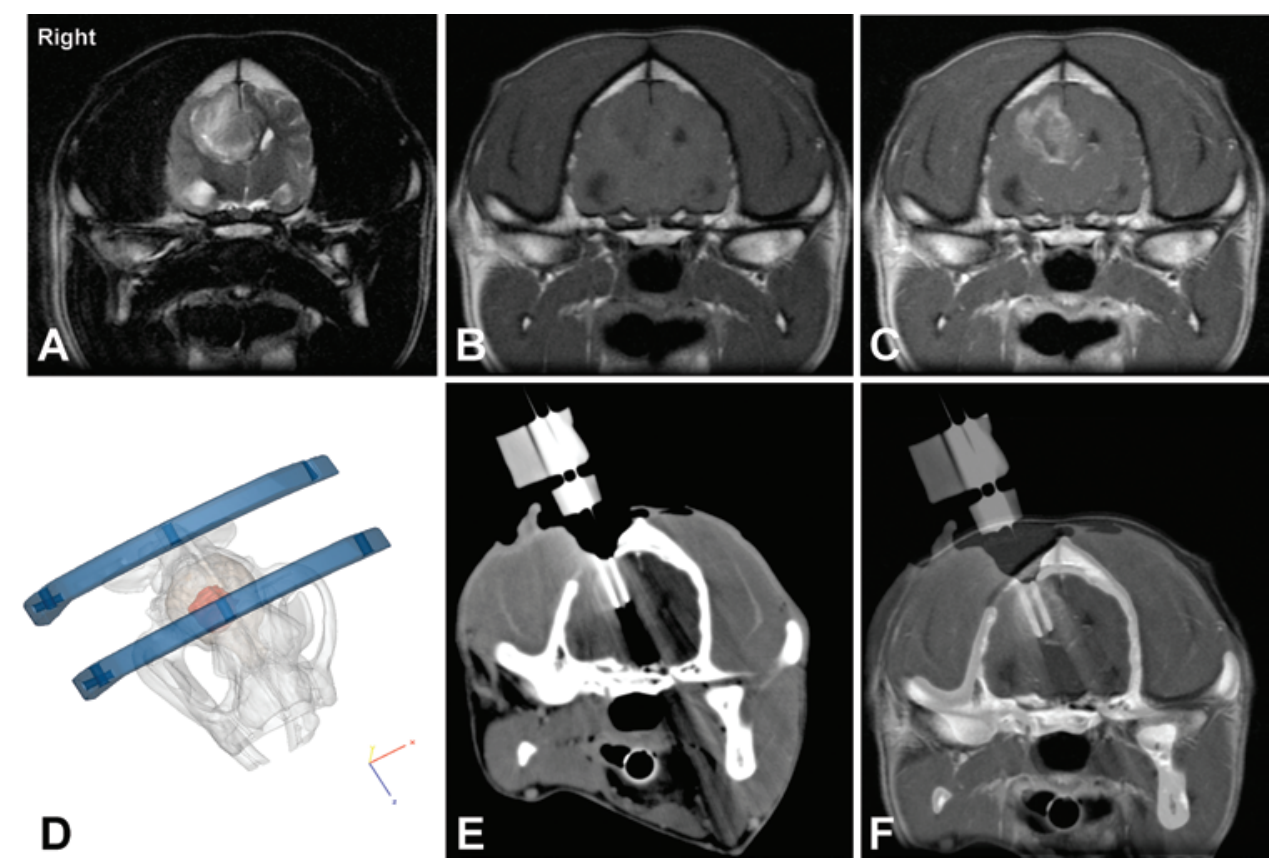

FIG. 3. Case 5: Stereotactic IRE treatment using an open craniectomy approach. Pretreatment T2-weighted (A), T1-weighted (B), and postcontrast T1-weighted (C) MR images demonstrating the GBM in the right frontoparietal region. 3D reconstructed image (D) showing the center-of-arc stereotactic apparatus and the skull, brain, and tumor (red). Intraoperative CT (E) and coregistered CT and MR (F) images with electrodes in situ within the tumor. The PDMS immobilization device affixed to the stereotactic frame is visible in $\mathbf{E}$ and $\mathbf{F}$. Figure is available in color online only. 
of interest (i.e., the area near the electrodes) that the electrically insulating boundaries at the edges of the domain do not significantly influence the results in the treatment zone.

The numerical models have been adapted to account for a dynamic nonlinear tissue conductivity that occurs as a result of electroporation and redistributes the electric field during the treatment. ${ }^{18,31,41}$ The significant nonlinear changes in the electrical conductivity of treated tissues occur because of cell membrane defects that facilitate the flow of ions and current through cells and are necessary to accurately represent IRE treatments. The dynamic conductivity, $\sigma(E, T)$, is a function of the electric field $(E)$ and temperature $(T)$ of the tissue during the IRE treatment. In tissue and tumors, this increase in conductivity is approximately 3-6 times the baseline conductivity when fully electroporated. ${ }^{22}$

Based on the tumor dimensions and computational simulations, IRE pulse parameters were determined to maximize coverage of the entire tumor volume and minimize damage to the surrounding healthy tissue for 5 of the 7 dogs. Specifically, tumors that were smaller than $2.5 \mathrm{~cm}^{3}$ were selected for entire coverage with up to 6 electrodes. In the cases of Dogs 2 and 5, corresponding to patients with glioblastoma multiforme (GBM), the tumors were larger than $2.5 \mathrm{~cm}^{3}$, so the treatment-planning strategy was to reduce the tumor volume as much as possible without affecting critical surrounding structures. We used an
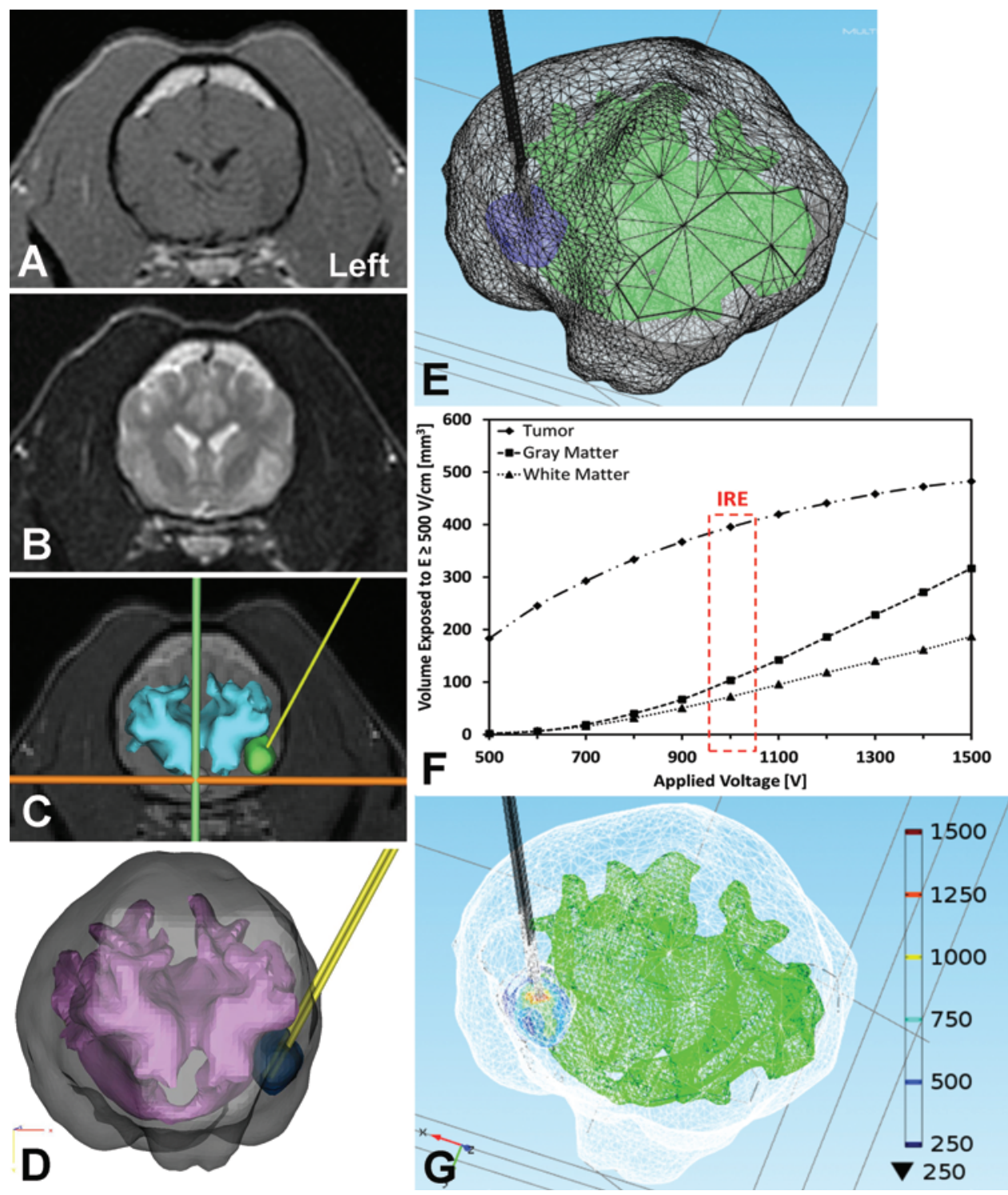

FIG. 4. Representative therapeutic planning procedure for IRE ablation of intracranial glioma with the NanoKnife system. Case 6: pretreatment postcontrast T1-weighted (A) and T2-weighted (B) axial MR images showing the nonenhancing Grade II oligodendroglioma in the left frontal lobe. C: Segmentation of the tumor (green), white matter (blue), and 2-electrode array probe trajectories (yellow) from 2D MR images. A 3D segmented composite model (D) was used to generate tetrahedral elemental volumetric mesh (E), which is imported into finite element analysis software. F: Parametric analysis output used to determine treated tumor volume. G: Simulated electrical field distribution $(\mathrm{V} / \mathrm{cm})$ in the final therapeutic plan. Figure is available in color online only. 
electric field threshold value of $500 \mathrm{~V} / \mathrm{cm}$ to computationally assess the coverage of the tumor and potential effects on the surrounding normal brain tissue. However, in the case of close proximity to critical structures such as the ventricles, we used $250 \mathrm{~V} / \mathrm{cm}$ as a conservative electric field threshold during the calculations. In Dog 6, for example, we developed a parametric study to compute the volumes of tumor tissue, gray matter, and white matter that would potentially be affected with different applied voltages using the $500 \mathrm{~V} / \mathrm{cm}$ threshold level (Fig. 4F). In this case, the IRE treatment was delivered at the 1000$\mathrm{V}$ level because it covered a significant fraction of the tumor while affecting a significantly smaller volume of gray matter and white matter in the surrounding tissue. A representative electric field distribution determined from these parameters can be seen in Fig. 4G, which shows the localized effect around the exposed electrodes within the tumor.

\section{Surgical Approaches}

Two surgical approaches were used for the IRE procedures. Dogs with a tumor associated with significant leptomeningeal contact, moderate-to-marked mass effect, or brain herniations or a tumor with geometry or size that necessitated insertion of $\geq 3$ sets of electrodes were treated with a rostrotentorial craniectomy of sufficient size to accommodate electrode array placement (Figs. 1 and 3). Dogs with deep-seated subcortical tumors associated with minimal mass effect or peritumoral edema were treated by using a bur-hole craniectomy and implantation of a probe guide pedestal (PGP) (Fig. 5). The PGP was constructed from threaded nylon rod, into which linear guide channels were machined to accommodate passage of 16-gauge sidecutting biopsy needles (Integra Radionics) and the IRE electrode probe configuration necessary to treat the dog's specific tumor. The PGP was anchored to the skull using titanium self-tapping bone screws (Quick Tap; Kinamed Inc.) and ultraviolet-curable urethane dimethacrylate gel (Triad Gel; Dentsply International, Inc.).

\section{Surgical Technique (Protocol Day 0)}

After induction of general anesthesia with IV propofol and fentanyl infusions, the dog was affixed to the stereotactic head frame, and the head was shaved and aseptically prepared for surgery. In the operating theater, each dog underwent a routine lateral rostrotentorial approach to
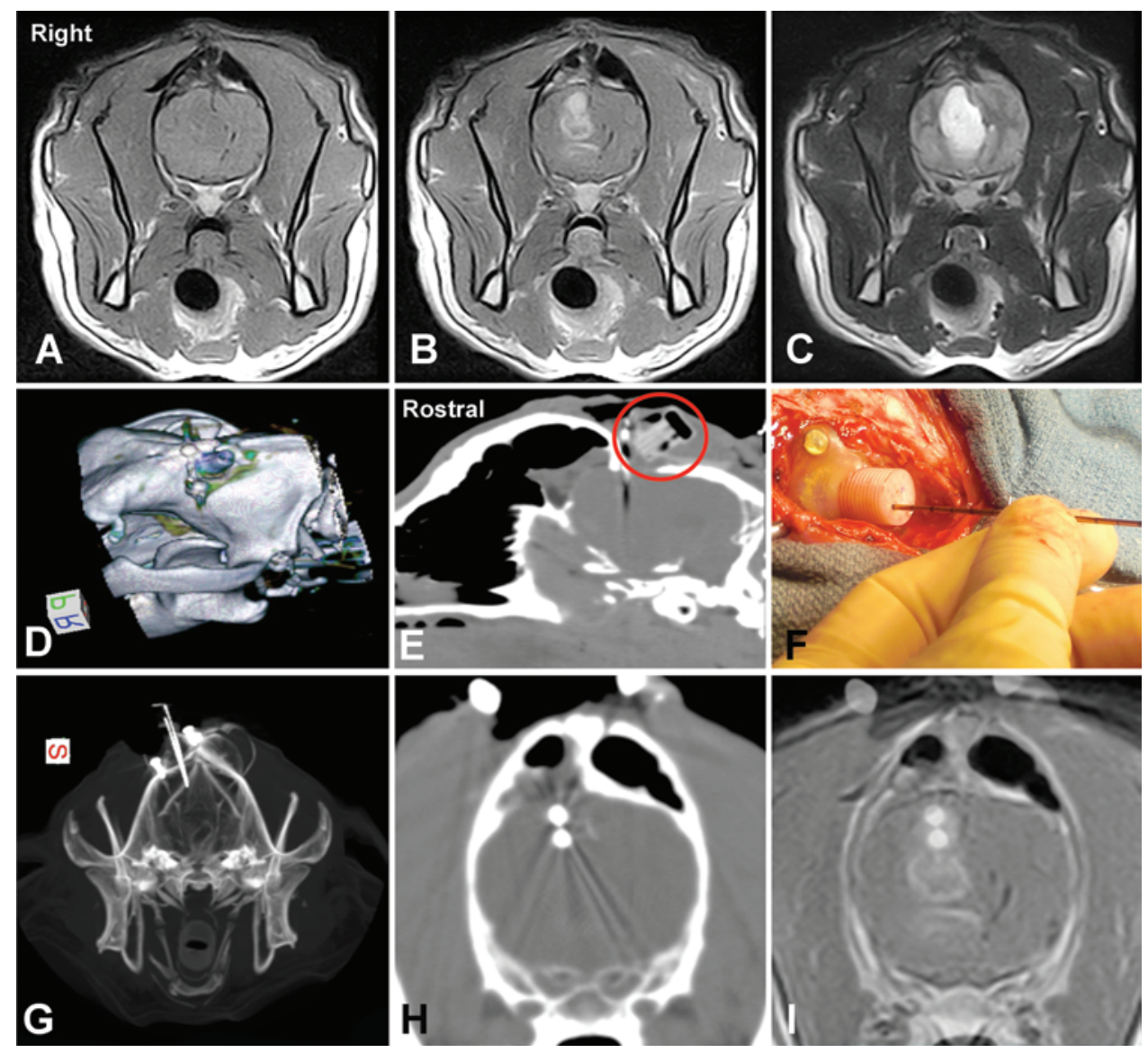

FIG. 5. Case 2: stereotactic IRE treatment using a PGP approach. Pretreatment T1-weighted (A), postcontrast T1-weighted (B), and T2-weighted (C) transverse MR images of a GBM in the right fronto-olfactory region. The implanted PGP can be seen in the surface-rendered reconstructed CT (D; blue), right parasagittal CT (E; red circle), and intraoperative (F) images. Shown also are intraoperative 3D reconstructed CT (G), CT (H), and coregistered CT and postcontrast T1-weighted MR (I) images of the electrodes in situ within the tumor. Figure is available in color online only. 
the skull. The final dog-specific approach (craniectomy vs PGP) to the tumor was performed, and brain biopsies were obtained by using the manipulator arm of the stereotactic head frame based on coordinates determined from the pretreatment plans. Before proceeding with the NanoKnife treatment, confirmation of viable glioma was verified by cytopathologic analysis of tissue sections performed by a veterinary pathologist. The tumor was histopathologically classified and graded according to World Health Organization criteria. ${ }^{28}$ After the biopsy, the surgical field was lavaged, covered with an impregnable, adhesive, iodinated barrier drape, and the dog was transported under anesthesia to the CT suite for IRE treatment. After positioning the dog on the CT couch, additional sterile draping was applied to the dog and CT unit. The electrode probes were then advanced into the target region either with fixation of the PDMS immobilization device to the stereotactic frame (Fig. 3) or through the implanted PGP (Fig. 5); probe placement was confirmed with CT.

Neuromuscular blocking agents (IV atracurium or IV rocuronium) were administered to effect 10 minutes before delivery of the IRE pulses. The tumor was then ablated according to the dog-specific treatment-planning protocols after activating the NanoKnife generator by depressing the footswitch. Pulse delivery was synchronized with the electrocardiogram (Cardiac Trigger Monitor 3000; Ivy Biomedical) to prevent the induction of any ventricular fibrillation or other cardiac arrhythmias. ${ }^{10}$

After the completion of IRE pulse delivery, the electrodes were removed from the brain, and precontrast and postcontrast CT scans of the brain were obtained. The dog was then transported back to the operating theater, where the surgical wound was lavaged and closed routinely. In those dogs in which a PGP was implanted, a solid nylon end cap was placed on the exposed proximal threads before closure. Each dog received perioperative antibiotics (IV cefazolin, $22 \mathrm{mg} / \mathrm{kg}$ every 8 hours) and buprenorphine (IV or SC, $0.03 \mathrm{mg} / \mathrm{kg}$ every 6-8 hours) for at least 36 hours after recovery from the IRE treatment.

\section{Caspase-3 Immunohistochemistry}

Formalin-fixed paraffin-embedded tumor samples were cut into 5- $\mu \mathrm{m}$ sections for caspase-3 immunohistochemistry. ${ }^{38}$ Antigen retrieval was performed with antigen-retrieval citrate buffer (citrate pH 6.0; caspase-3, Dako 3-in1 ) by boiling for 10 minutes. The sections were washed in neutral phosphate-buffered saline (PBS) for 5 minutes after antigen retrieval. Endogenous peroxidase activity was quenched by exposing the sections to $0.3 \%$ hydrogen peroxide in methanol for 30 minutes at room temperature. After blocking with $10 \%$ normal goat serum (Protein Block; Dako) for 30 minutes at $37^{\circ} \mathrm{C}$, the sections were incubated with rabbit polyclonal caspase-3 (1:60; Abcam) in $0.01 \mathrm{M}$ PBS containing $1 \%$ normal goat serum and $0.3 \%$ Triton $\mathrm{X}-100$ for 30 minutes at $20^{\circ} \mathrm{C}$. The sections were then rinsed with Tris-buffered saline and incubated for 1 hour with biotinylated anti-rabbit IgG (1:200, ABC kit; Vector Laboratories) in $0.01 \mathrm{M}$ PBS containing $1 \%$ normal goat serum and $0.3 \%$ Triton X-100. Subsequently, the sections were rinsed with Tris-buffered saline and incubated with an avidin-biotin-horseradish peroxidase complex (1:200,
$\mathrm{ABC}$ kit) for 1 hour at $37^{\circ} \mathrm{C}$. Visualization occurred via the alkaline phosphatase method using a fast red chromogen and hematoxylin counterstain. Canine tonsil was used as a positive control. Negative control sections received identical treatment except they were not exposed to the primary antibodies.

\section{Caspase-3 and Poly(ADP-Ribose) Polymerase Western Blotting}

Brain tumor proteins were prepared by extraction in neutral (pH 7.4) Tris containing 0.1\% Triton X-100. Protein concentrations were determined by using a bicinchoninic acid protein assay (Sigma-Aldrich). From each sample, $50 \mu \mathrm{g}$ of protein was separated on a $12 \%$ polyacrylamide gel. After electrophoresis, protein was transferred to a nitrocellulose membrane that was blocked at room temperature for 1 hour in a solution of $1 \times$ Tris-buffered saline containing 5\% skim milk, $1 \%$ bovine serum albumin, and $0.05 \%$ Triton $\mathrm{X}-100$. The membrane was then incubated overnight at $4{ }^{\circ} \mathrm{C}$ in the blocking solution containing rabbit antiactive and procaspase 3 polyclonal (1:1000; Abcam) and rabbit polyclonal anti-poly(ADP-ribose) polymerase (1:1500; Abcam) antibodies. Membranes were washed and then probed with horseradish-peroxidase-conjugated goat anti-rabbit secondary antibody (1:1000; BD Bioscience), and detection was performed by using a chemiluminescence detection kit (GE Healthcare Life Sciences). The positive control consisted of a canine J3T glioma cell line incubated for 24 hours in $1 \mu \mathrm{M}$ staurosporine in dimethyl sulfoxide. Rabbit anti- $\beta$-actin polyclonal antibody (BioVision) was used as an equivalent protein loading control.

\section{Results \\ Clinicopathologic Characteristics}

Seven dogs with spontaneous intracranial gliomas (Table 1) were treated. The IRE treatment in Case 1 was described previously. ${ }^{16}$ The mean \pm SD age of the dogs was $9.3 \pm 1.6$ years, and the mean pretreatment tumor volume (according to T2-weighted imaging) was $1.9 \pm 1.4 \mathrm{~cm}^{3}$. Each dog had structural tumor-associated epilepsy and was receiving 1 or more anticonvulsants and corticosteroids at the time of enrollment. Each dog also had interictal neurological deficits consistent with the neuroanatomical location of the tumor, including behavioral change $(\mathrm{n}=$ 3 ), ipsilateral circling $(n=3)$, homonymous hemianopia ( $=2$ ), obtundation $(\mathrm{n}=2)$, and hemi-inattention $(\mathrm{n}=1)$. The median pretreatment KPS score was 70 (range 30-75). The median duration of clinical signs before IRE treatment was 26 days (range 19-188 days).

\section{NanoKnife IRE Treatment}

The surgical approaches, treatment parameters derived from therapeutic plans, and voltages and currents measured during treatment of the 7 dogs are summarized in Tables 2 and 3 . The median total time required for pulse delivery, calculated from final electrode probe placement into the target until withdrawal of the probes from the brain, was 9 minutes (range 4-34 minutes). No alterations in the electrocardiogram were noted during pulse delivery. In 6 of the 7 dogs, IRE treatment was executed without com- 

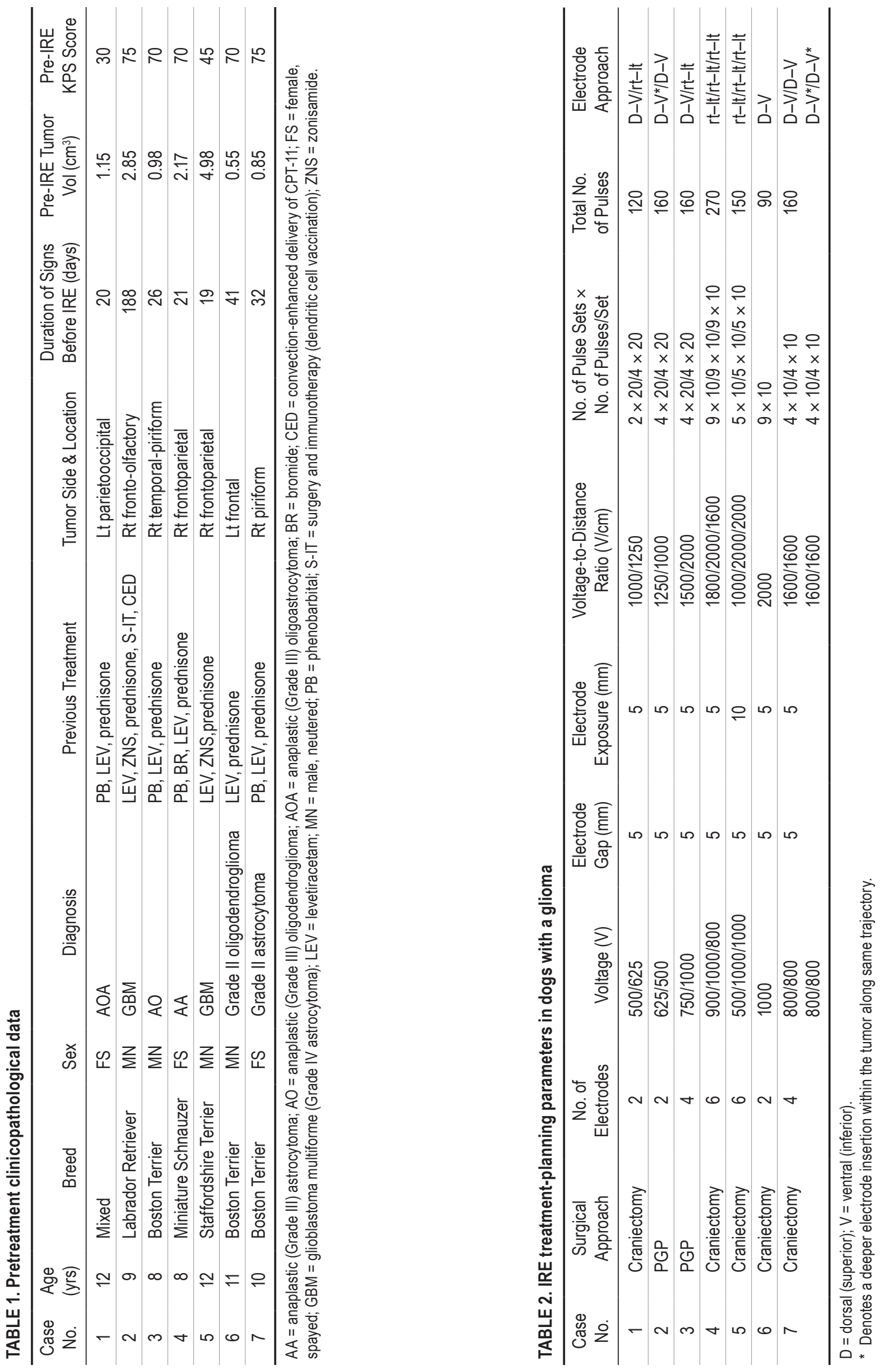
TABLE 3. Currents and voltages measured during IRE treatment of canine glioma

\begin{tabular}{|c|c|c|c|c|c|c|c|}
\hline $\begin{array}{l}\text { Case } \\
\text { No. }\end{array}$ & Electrode Orientation & $\begin{array}{l}\text { Electrode } \\
\text { Gap (mm) }\end{array}$ & $\begin{array}{c}\text { Electrode } \\
\text { Exposure }(\mathrm{mm})\end{array}$ & $\begin{array}{l}\text { Voltage } \\
\text { (V) }\end{array}$ & $\begin{array}{l}\text { Voltage-to-Distance } \\
\text { Ratio }(\mathrm{V} / \mathrm{cm})\end{array}$ & $\begin{array}{l}\text { Current } \\
\text { (A) }\end{array}$ & $\begin{array}{l}\text { No. of } \\
\text { Pulses }\end{array}$ \\
\hline \multirow[t]{2}{*}{1} & Parallel & 5 & 5 & 477 & 954 & 1.3 & 40 \\
\hline & Perpendicular & 5 & 5 & 605 & 1210 & 1.4 & 80 \\
\hline \multirow[t]{2}{*}{2} & Deep & 5 & 5 & 514 & 1028 & 2.1 & 80 \\
\hline & Superficial & 5 & 5 & 627 & 1254 & 3.2 & 80 \\
\hline \multirow[t]{2}{*}{3} & Parallel & 5 & 5 & 768 & 1536 & 2.1 & 80 \\
\hline & Perpendicular & 5 & 5 & 1037 & 2074 & 2.1 & 80 \\
\hline \multirow[t]{3}{*}{4} & 6-electrode array & 5 & 5 & 830 & 1660 & 1.9 & 90 \\
\hline & & 5 & 5 & 927 & 1854 & 2.1 & 90 \\
\hline & & 5 & 5 & 1010 & 2020 & 1.7 & 90 \\
\hline \multirow[t]{5}{*}{5} & Original & 5 & 10 & 512 & 1024 & 4.0 & 10 \\
\hline & 6-electrode array & 5 & 10 & 1140 & 2280 & 17.0 & 10 \\
\hline & Revised & 5 & 10 & 512 & 1024 & 4.0 & 50 \\
\hline & 6-electrode array & 5 & 10 & 955 & 1910 & 13.0 & 50 \\
\hline & & 5 & 10 & 955 & 1910 & 11.0 & 50 \\
\hline 6 & 2-electrode array & 5 & 5 & 1024 & 2048 & 3.4 & 90 \\
\hline \multirow[t]{4}{*}{7} & Deep & 5 & 5 & 840 & 1680 & 1.7 & 40 \\
\hline & 4-electrode array & 5 & 5 & 840 & 1680 & 1.5 & 40 \\
\hline & Superficial & 5 & 5 & 840 & 1680 & 1.7 & 40 \\
\hline & 4-electrode array & 5 & 5 & 840 & 1680 & 1.5 & 40 \\
\hline
\end{tabular}

plications, and the actual energy delivered to the tumor, as determined from the voltage and current output data from the NanoKnife, was representative of intended energy delivery from the therapeutic plans (Tables 2 and 3). In Case 5 (Fig. 3), there was suspicion of invasion of the GBM into the lateral ventricle and the potential for increased current delivery associated with pulsing in the conductive cerebrospinal fluid medium. Recording of high currents after delivery of the first two 10-pulse sequences (Table 3, original treatment) confirmed this suspicion, which resulted in manual abortion of pulse delivery and necessitated intraoperative revision of pulse-delivery parameters to reduce the current (Table 3, revised treatment). Intraoperative and acute postoperative imaging studies indicated that, except for in Case 5, IRE treatment was achieved without inducing or exacerbating edema or significant hemorrhage from pulse delivery (Fig. 6).

\section{Adverse Events}

All adverse events observed within the 14-day posttreatment observation are reported in Table 4. Overall, 2 of the 7 dogs developed significant clinical toxicity within 14 days of the IRE treatment. Dog 2 developed fatal (Grade 5) aspiration pneumonia. Dog 5 developed cerebral edema associated with a 15-point decline in the KPS score and a transient exacerbation of preexisting neurological deficits that were managed effectively with diuretics and corticosteroids. No surgical site or CNS infections were observed over the entire study period. Mild-to-moderate (CTCAE Grades 1 and 2; Table 4) adverse events were observed in every dog during the 14-day posttreatment safety evaluation period. Grade 1 and 2 adverse events included acute posttreatment seizures in 5 of the 7 dogs, perioperative nausea, vomiting, or diarrhea in $4 \mathrm{dogs}$, and aspiration in $1 \mathrm{dog}$.

Adverse events were observed in 5 of 6 dogs between
Days 15 and 365 of the study period and are presented in Table 5. Severe adverse events were noted in 2 of 6 dogs and mild-to-moderate adverse events in 3 of 6 dogs. Neurological adverse events occurred in 4 of 6 dogs, including seizures in 4 dogs and CNS necrosis and leukoencephalopathy in $1 \mathrm{dog} .{ }^{16}$

\section{Clinical Outcomes}

Tables 6 and 7 summarize the clinical outcomes over the study period. Within 1 hour of recovery from anesthesia after treatment, 6 of the 7 dogs were alert and capable of assuming sternal recumbency, and 4 of 5 dogs that were capable of unassisted ambulation preoperatively were able to ambulate within 4 hours of recovery. The median duration of hospitalization for IRE therapy, inclusive of the pretreatment planning, was 4 days (range 3-11 days). Among the dogs that survived to discharge, the KPS scores were improved over the pretreatment values in 5 of 6 and 6 of 6 dogs at 7 and 14 days after treatment, respectively (Tables 1 and 6). Seizure control improved (Engel Classes I-III; Table 7) in 5 of the 6 dogs that survived to discharge, with 2 of 6 dogs demonstrating seizure freedom (Engel Class I) on Days 15-365 of the study.

The median survival in this group of dogs was 119 days (range 1 to $>940$ days; Table 6). Two dogs underwent additional therapies after completing the initial mandatory 14-day post-IRE observation (Table 7). Of the 6 dogs that survived the initial 14-day safety evaluation window, 2 completed the prescribed 12-month follow-up period (Table 7). Each of the remaining 4 dogs was humanely killed during the study follow-up period at the request of the owner; in 3 of these dogs, RANO criteria of PD were documented in proximity to death (Table 7). When requested by the owner, dogs were killed humanely a median of 3 days (range 1-17 days) from the time of documented PD. 
T1

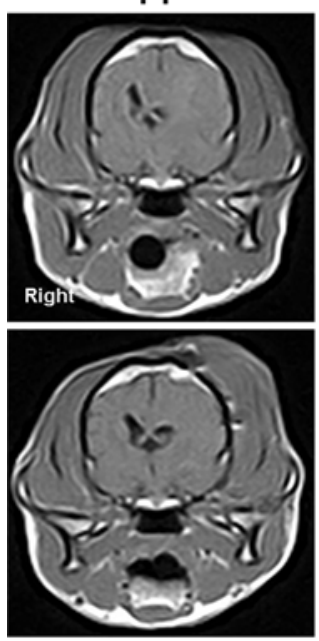

T1

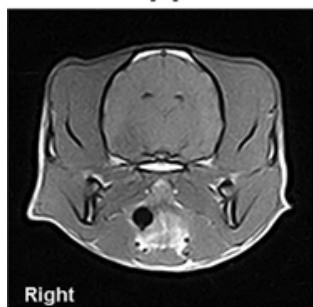

ren

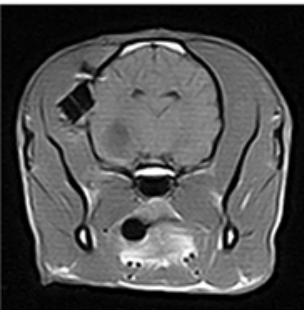

$\mathrm{T} 1+\mathrm{Gd}$
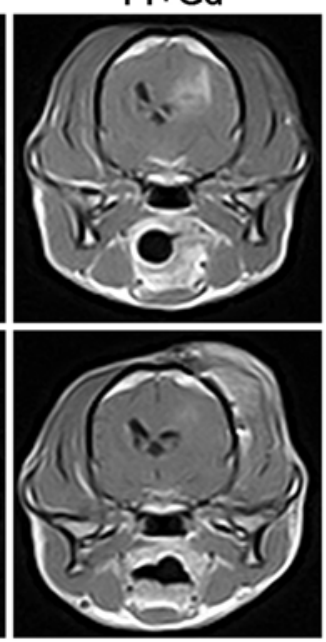

$\mathrm{T} 1+\mathrm{Gd}$
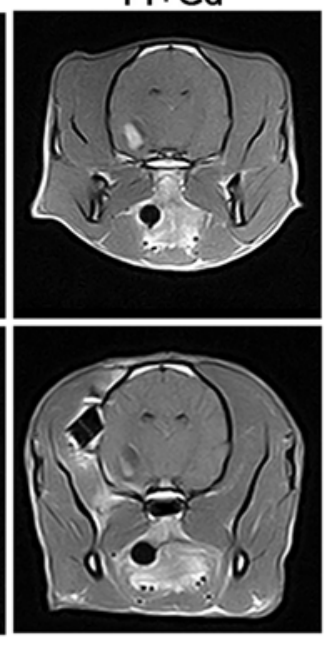

\section{Post-IRE}

1 week
T2
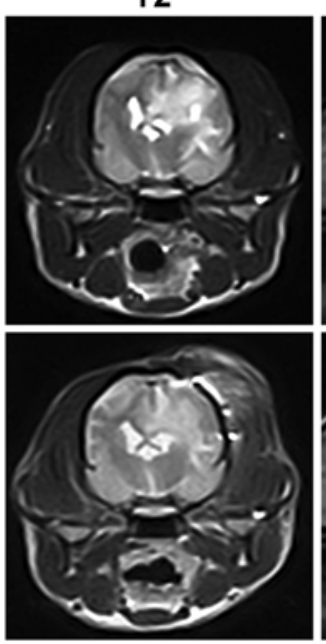

$\mathrm{T} 1+\mathrm{Gd}$
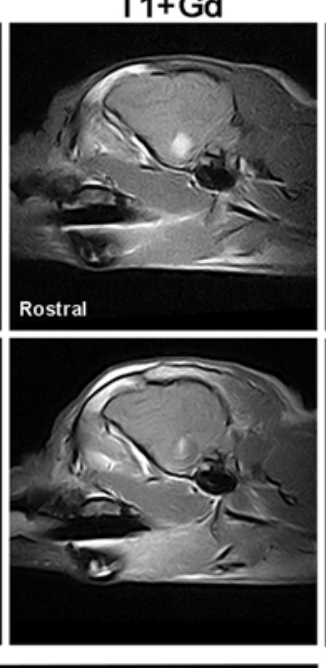

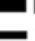

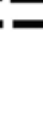

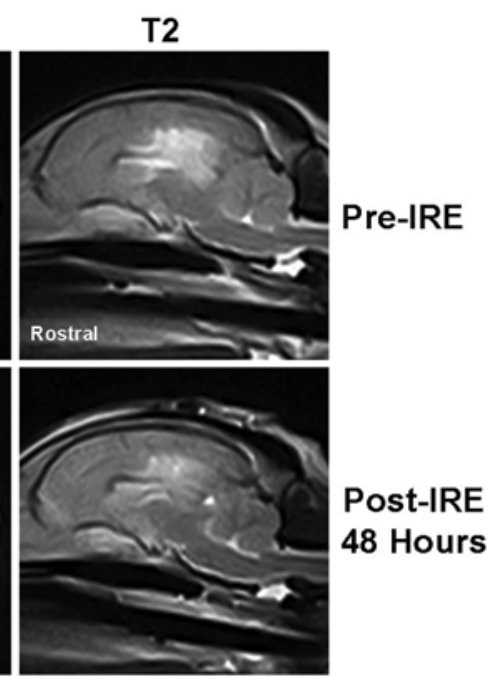

Post-contrast CT

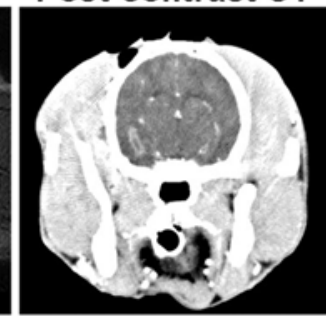

Pre-IRE

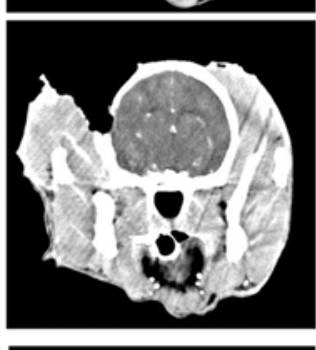

Post-IRE

15 minutes

FIG. 6. Acute posttreatment imaging-based therapeutic response evaluations. Case 1: pretreatment (upper panels, top row) and 48-hour posttreatment (upper panels, bottom row) MRI examinations. There is severe edema and mass effect present on the pretreatment images. After treatment, there is a reduction in both the contrast-enhancing tumor burden and peritumoral edema. Case 3: pretreatment MR and postcontrast CT (lower panels, top row) and corresponding posttreatment (lower panels, bottom row) images. A reduction in the enhancing portion of the tumor can be seen on the immediate posttreatment CT and MR images 1 week after IRE treatment. The implanted PGP is visible in the posttreatment MR images.

TABLE 4. Adverse events that occurred within 14 days of IRE treatment of canine intracranial glioma

\begin{tabular}{|c|c|c|c|c|}
\hline \multirow{2}{*}{$\begin{array}{l}\text { Case } \\
\text { No. }\end{array}$} & \multirow{2}{*}{$\begin{array}{c}\text { Intraoperative Adverse Event }^{*} \\
\text { Nervous SOC/Grade }\end{array}$} & \multicolumn{3}{|c|}{ Posttreatment Adverse Event ${ }^{\star}$} \\
\hline & & Nervous SOC/Grade & Respiratory SOC/Grade & Gastrointestinal SOC/Grade \\
\hline 1 & None & Seizure/1 & Aspiration/2 & None \\
\hline 2 & None & Seizure/2 & Aspiration/5 & Vomiting/2 \\
\hline 3 & None & None & None & Diarrhea/1 \\
\hline 4 & Intracranial hemorrhage (biopsy)/1 & Seizure/2 & None & None \\
\hline 5 & Cerebral edema/3 & None & None & Vomiting/2 \\
\hline 6 & None & Seizure/1 & None & $\begin{array}{l}\text { Vomiting/1 } \\
\text { Diarrhea/1 }\end{array}$ \\
\hline 7 & None & Seizure/2 & None & None \\
\hline
\end{tabular}

SOC = system organ class.

* Classified and graded according to the Cancer Therapy Evaluation Program's CTCAE. 
TABLE 5. Adverse events that occurred 15-365 days after IRE treatment of canine intracranial glioma

\begin{tabular}{cl}
\hline Case No. & Adverse Event/Grade* \\
\hline 1 & $\begin{array}{l}\text { CNS necrosis/4 } \\
\text { Leukoencephalopathy/3 } \\
\text { Seizure/1 }\end{array}$ \\
& Seizure/2 \\
Vomiting \&/or diarrhea 1 \\
\hline 3 & None \\
\hline 5 & Seizure/2 \\
& Vomiting \&/or diarrhea 1 \\
\hline 6 & Rash maculopapular/1 \\
& Heart failure/2 \\
\hline 7 & Vomiting \&/or diarrhea/2 \\
& Pancreatitis/3 \\
& Seizure/3 \\
\hline
\end{tabular}

* Classified and graded according to the Cancer Therapy Evaluation Program's CTCAE.

\section{Objective Therapeutic Response Assessments}

In cases with a contrast-enhancing tumor, a reduction in the enhancing tumor burden was apparent on acute posttreatment imaging examinations (Fig. 6). Dogs 6 and 7 (Table 7) had nonenhancing isoattenuating (nontarget) low-grade gliomas that were difficult to differentiate from surrounding brain on CT images; thus, CT-based therapeutic responses were not evaluated in these cases.

Four dogs had posttreatment tumor biopsies performed 1-2 hours after IRE treatment during closure of the surgical wound in the operating theater (Dogs 3,4, and 5) or at the time of necropsy within 24 hours (Dog 2) of IRE treatment. In each dog in which a posttreatment biopsy was performed, samples revealed that IRE ablation was associated with complete obliteration of cytoarchitectural features of the tumor (Figs. 7 and 8). Among the amorphous eosinophilic background, remaining recognizable cells frequently demonstrated morphological features of apoptosis, including nuclear pyknosis and karyorrhexis, intense cytoplasmic eosinophilia, apoptotic body formation, and caspase-3 immunoreactivity. IRE-induced tumor apoptosis was also demonstrated by the cleavage of procaspase- 3 and poly(ADP-ribose) polymerase seen on Western blots (Fig. 7). As determined from the necropsy of Dog 2, IRE treatment was associated with a sharply defined submillimeter line of demarcation between the ablated tumor and the adjacent brain, with a transitional zone of vacuolated neural tissue and perivascular inflammation interposed between the microscopically normal-appearing neuropil and the treated tumor (Fig. 8).

Objective therapeutic responses determined from follow-up MRI examinations using RANO criteria are summarized in Table 7 and Fig. 9. Overall, 80\% (4 of 5) of dogs in this study with a quantifiable target lesion demonstrated an objective response to IRE treatment. Dog 2 also had a target lesion that met RANO criteria, but he died before the performance of posttreatment MRI; tumor ablation was confirmed at necropsy (Figs. 7 and 8).

\section{Discussion}

\section{Procedural Observations}

As has been shown in studies of IRE for ablation of tumors in other organs, $, 7,29,32$ IRE was generally a safe and well-tolerated treatment in this study. In the absence of severe complications, most of the dogs were able to be discharged from the hospital within 72 hours of treatment, and all dogs that survived to discharge had 14-day KPS scores that were improved over the pretreatment value. In addition, 4 of the 5 dogs with a target lesion that met RANO criteria demonstrated an objective reduction in contrast-enhancing tumor burden after treatment, which was accompanied by clinical improvements in tumorassociated neurological dysfunction. Although we and others previously observed treatment-induced cerebral edema in normal canine and rodent brains ablated with IRE, $13,19,21$ we postulate that the rapid reduction in tumor volume associated with IRE ablation provides a plausible explanation for both the acute clinical improvements and lack of significant edema observed in 6 of the 7 dogs in this study, but we acknowledge that aggressive antiedema

TABLE 6. Clinical outcomes for dogs with glioma treated with IRE

\begin{tabular}{|c|c|c|c|c|c|c|c|}
\hline \multirow{2}{*}{$\begin{array}{l}\text { Case } \\
\text { No. }\end{array}$} & \multirow{2}{*}{$\begin{array}{c}\text { Duration of } \\
\text { Hospitalization (days) }^{*}\end{array}$} & \multicolumn{2}{|c|}{ Edema Score $\dagger$} & \multicolumn{3}{|c|}{ KPS Score Change } & \multirow{2}{*}{$\begin{array}{l}\text { Survival After } \\
\text { IRE (days) }\end{array}$} \\
\hline & & Pretreatment & Posttreatment & Discharge & Day 7 & Day 14 & \\
\hline 1 & 10 & 2 & 1 & 25 & 25 & 30 & 147 \\
\hline 2 & 3 & 1 & 1 & ND & ND & ND & 1 \\
\hline 3 & 4 & 0 & 0 & 10 & 10 & 20 & 129 \\
\hline 4 & 5 & 1 & 0 & 10 & 10 & 10 & $>940$ \\
\hline 5 & 11 & 1 & 2 & 15 & -10 & 15 & 96 \\
\hline 6 & 3 & 1 & 1 & 10 & 10 & 15 & 425 \\
\hline 7 & 3 & 1 & 1 & 5 & 5 & 10 & 67 \\
\hline
\end{tabular}

$\mathrm{ND}=$ no data (the dog died before discharge).

* Includes 1 day for therapeutic planning.

$\dagger 0$, no detectable edema; 1 , moderate peritumoral edema or mass effect with no (or improved) shift; 2 , severe regional edema with shift or mass effect (or worsening of shift/mass effect).

$\ddagger$ Relative to pretreatment value. 
TABLE 7. Objective therapeutic response assessments in dogs with glioma

\begin{tabular}{|c|c|c|c|c|c|c|c|c|c|c|c|}
\hline $\begin{array}{l}\text { Case } \\
\text { No. }\end{array}$ & $\begin{array}{c}\text { Other Post-IRE } \\
\text { Therapy }\end{array}$ & $\begin{array}{c}\text { Response } \\
\text { Criteria }\end{array}$ & $\begin{array}{c}\text { RANO } \\
\text { Target Lesion }\end{array}$ & $2 \mathrm{Mos}$ & 3 Mos & 4 Mos & $5 \mathrm{Mos}$ & $6 \mathrm{Mos}$ & 9 Mos & 12 Mos & $\begin{array}{l}\text { Engel } \\
\text { Class }\end{array}$ \\
\hline 1 & RT & $\begin{array}{l}\text { RANO } \\
\text { KPS score }\end{array}$ & Yes & $\begin{array}{l}\text { ND } \\
70\end{array}$ & $\begin{array}{l}\text { ND } \\
70\end{array}$ & $\begin{array}{l}\text { CR } \\
70\end{array}$ & $\begin{array}{l}P^{*} \\
55\end{array}$ & Killed $†$ & & & III \\
\hline 3 & ECT & $\begin{array}{l}\text { RANO } \\
\text { KPS score }\end{array}$ & Yes & $\begin{array}{l}\text { PR } \\
90\end{array}$ & $\begin{array}{l}\text { PR } \\
90\end{array}$ & $\begin{array}{l}\text { PD } \ddagger \\
70\end{array}$ & Killed $\dagger$ & & & & III \\
\hline 4 & None & $\begin{array}{l}\text { RANO } \\
\text { KPS score }\end{array}$ & Yes & $\begin{array}{l}\text { ND } \\
90\end{array}$ & $\begin{array}{l}\text { CR } \\
90\end{array}$ & $\begin{array}{l}\text { ND } \\
100\end{array}$ & $\begin{array}{l}\text { ND } \\
\text { ND }\end{array}$ & $\begin{array}{l}\text { CR } \\
100\end{array}$ & $\begin{array}{l}\text { CR } \\
100\end{array}$ & $\begin{array}{l}\text { CR } \\
100\end{array}$ & I \\
\hline 5 & None & $\begin{array}{l}\text { RANO } \\
\text { KPS score }\end{array}$ & Yes & $\begin{array}{l}\text { PR } \\
75\end{array}$ & $\begin{array}{l}\text { PD } \ddagger \\
40\end{array}$ & Killed† & & & & & III \\
\hline 6 & None & $\begin{array}{l}\text { RANO } \\
\text { KPS score }\end{array}$ & No & $\begin{array}{l}\text { ND } \\
90\end{array}$ & $\begin{array}{l}\mathrm{SD} \\
90\end{array}$ & $\begin{array}{l}\text { ND } \\
\text { ND }\end{array}$ & $\begin{array}{l}\text { ND } \\
\text { ND }\end{array}$ & $\begin{array}{l}S D \\
90\end{array}$ & $\begin{array}{l}S D \\
90\end{array}$ & $\begin{array}{l}S D \\
90\end{array}$ & I \\
\hline 7 & None & $\begin{array}{l}\text { RANO } \\
\text { KPS score }\end{array}$ & No & $\begin{array}{l}\text { SD } \\
95\end{array}$ & Killed $†$ & & & & & & IV \\
\hline
\end{tabular}

$\mathrm{CR}=$ complete response; $\mathrm{ECT}=$ bleomycin electrochemotherapy (performed after documented PD); ND = no data available; $\mathrm{PR}=$ partial response; $\mathrm{RT}=$ fractionated radiotherapy (initiated 16 days after IRE; total dose 50 Gy in 20 2.5-Gy fractions).

* Treatment-related brain necrosis and leukoencephalopathy confirmed at necropsy.

$\dagger$ The dog was killed humanely at the request of the owner.

$\ddagger$ New lesions distant from original tumor/treatment site.

prophylaxis may also have contributed to the mitigation of procedural edema. The rapid reduction in tumor mass and short duration of hospitalization experienced by several of the dogs shows the potential for the NanoKnife to be combined with other adjuvant therapies in the acute postoperative period. In addition, IRE ablation may provide an alternative means of minimally invasive and expedient local tumor control when other methods, such as stereotactic radiosurgery, carry unacceptable risks or have failed.

The decision to perform posttreatment tumor biopsies arose from ambiguity in the literature regarding the mechanism of IRE-induced cell death and the need to evaluate the validity of contrast-enhanced imaging techniques as acceptable surrogates of objective tumor response to IRE treatment of gliomas. Cellular death induced by pulsed electrical fields has been reported to occur via both necrosis and apoptosis in other normal and neoplastic tissues, including brain tissue. ${ }^{4,13,33,38}$ Reports of investigations in which pathologic evaluations of IRE ablated spontaneous soft-tissue tumors were performed in the acute posttreatment setting have principally described morphological features of necrosis. ${ }^{34,46}$ Given that reports of previous investigations in rodent and canine brain models indicate that electroporation is capable of transiently inducing disruption of the blood-brain barrier in the treated region over a wide range of applied electrical field strengths, corresponding to gadolinium contrast enhancement in electroporated regions of the brain, ${ }^{13,19,21}$ we were unsure of the use of contrast-enhanced imaging techniques as an objective measure of tumor response. It has also been shown that microvascular flow is disrupted transiently in proximity to the delivery of electroporation pulses, which could result in an artifactual reduction in tumor enhancement unassociated with cell death, similar to a pseudoresponse. ${ }^{18,37} \mathrm{Re}-$
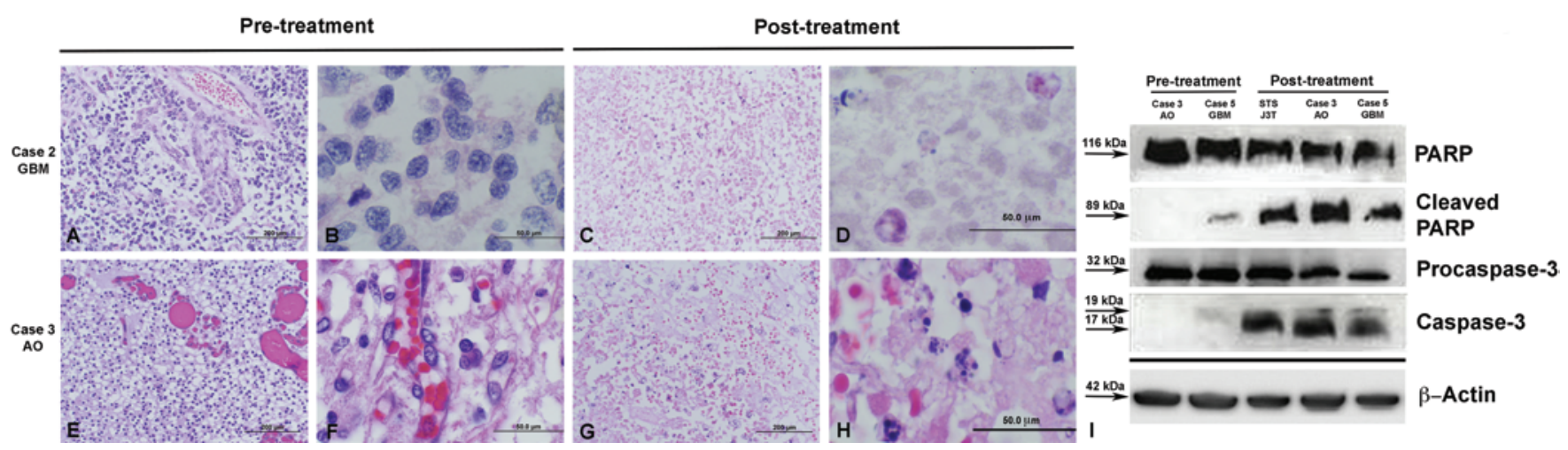

FIG. 7. Pathology of canine gliomas treated with IRE: photomicrographs of pretreatment tumor biopsy specimens from Cases 2 and $3(\mathrm{H} \& \mathrm{E}[\mathbf{A}, \mathbf{E}$, and $\mathbf{F}]$ and anti-caspase-3 [B] stains). Treated regions showed complete obliteration of cytoarchitectural features (C and G [H \& E stain]), with remaining cells showing immunoreactivity to caspase-3 (D) and morphological features of apoptosis (H [H \& E stain]). I: IRE-induced glioma apoptosis is evident on immunoblots from Cases 3 and 5 as indicated by cleavage of procaspase-3 and poly(ADP-ribose) polymerase (PARP) after treatment. AO = anaplastic oligodendroglioma; STS-J3T = staurosporine-treated canine glioma cell line J3T (positive control). Figure is available in color online only. 

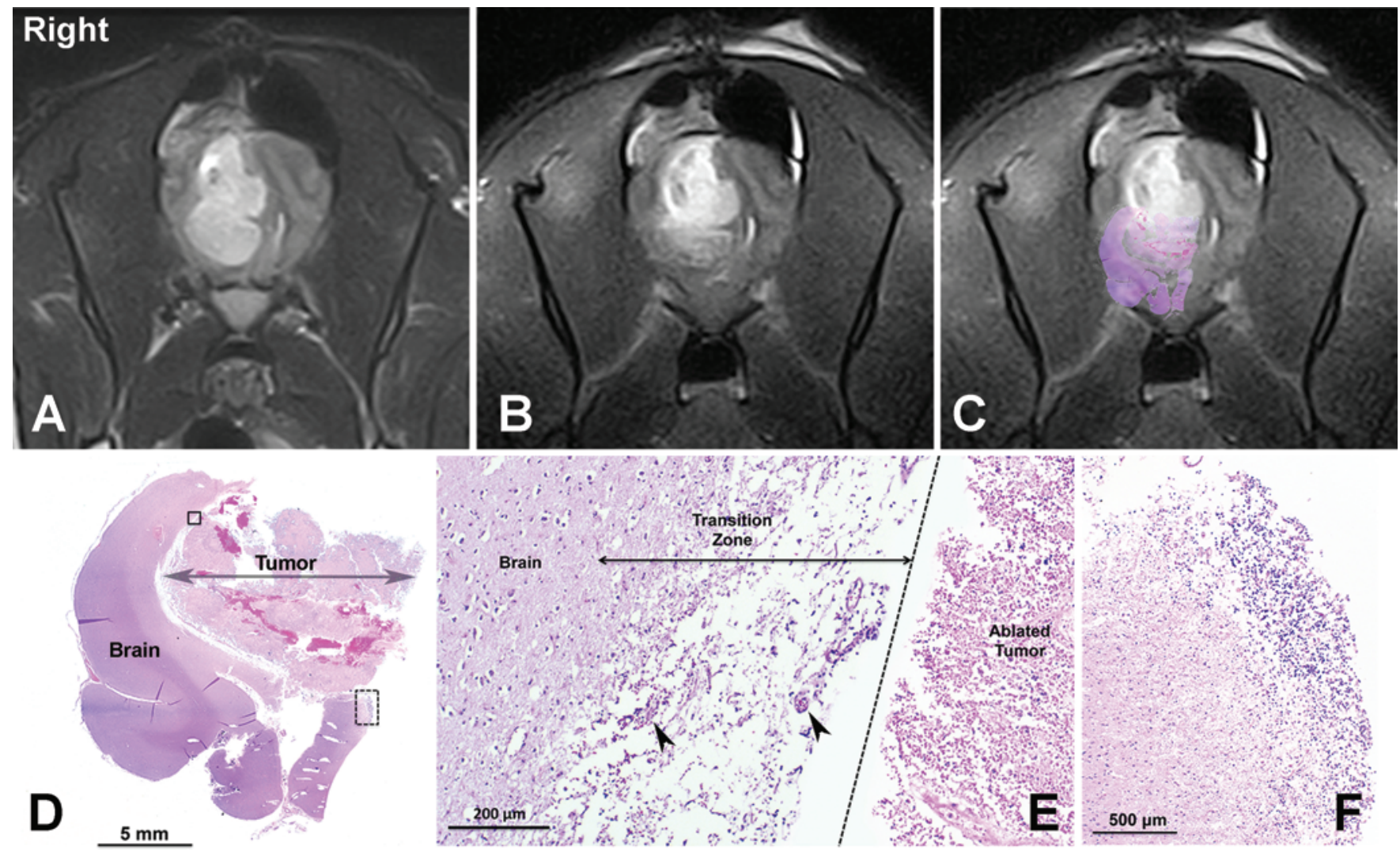

FIG. 8. Case 2: acute pathologic effects of IRE ablation in the brain. A: Pretreatment, transverse T2-weighted MR image showing the GBM in the right frontal lobe. B: Immediate postmortem posttreatment T2-weighted MR image obtained at the same level of the frontal lobe as that in A. C: Merged postmortem posttreatment T2-weighted MR image (B) with subgross brain slice (D) obtained at necropsy. The multifocal punctate regions of hemorrhage in the treated region correspond to the T2 hypointense areas shown in B. D: Subgross brain slice obtained from the ventral aspect of the frontal lobe showing the sharp delineation between ablated tumor and surrounding brain (H \& E stain). E: Microscopic effects of IRE ablation at the tumor-brain tissue interface (solid inset, D). The ablated tumor appears as an intensely eosinophilic region on the right of the image. A transition zone containing vacuolated neuropil and perivascular inflammatory cuffs (arrowheads) can be seen between the ablated tumor and the adjacent brain tissue. F: A microscopic focus of viable neoplastic cells (broken inset, D) exists outside of the treated region (H \& E stain). Figure is available in color online only.

sults from posttreatment imaging studies and biopsies suggest that using RANO criteria is appropriate for assessing therapeutic responses to IRE and show that the reduction in contrast-enhancing tumor after IRE ablation resulted from tumor death.

The dog-specific imaging-based therapeutic planning that was performed before IRE treatment was crucial for selecting the electrical parameters, electrode probe location, and approach that would enable us to maximize tumor coverage and minimize potential damage to the surrounding normal brain tissue. Other than our initial in vivo studies quantifying the extent of the IRE zone of ablation in normal canine brain, there are no experimental data quantifying the effects of the pulsed electric fields in brain tumor tissue. ${ }^{13,18}$ By using computational models that incorporate the anatomical and electrical tissue heterogeneities (e.g., tumor, white matter, gray matter), we mitigated the trial-and-error experimental requirement for determining the pulse parameters. The models we developed include the electrical and thermal responses of tissue to IRE therapy to prevent undesired lethal effects in critical regions that are in close proximity to the treatment zone. ${ }^{17,18}$ Perhaps the most important advantage of this technique is that the main parameter that determines the success of the IRE therapy is the electric field distribution, which can be predicted with our computational models.

\section{Adverse Effects}

One dog (Case 5) experienced severe direct neurotoxicity in the form of cerebral edema associated with the IRE procedure. This dog demonstrated an immediate but transient deterioration in preoperative neurological deficits after recovery. It should be noted that this dog received an unexpectedly high energy dose as a result of pulse delivery to the ventricular system and the increased conductivity of cerebrospinal fluid compared with that of tumor tissue. The edema was managed effectively with corticosteroids and diuretics, and the dog's KPS score ultimately improved over the pretreatment value by 14 days after treatment. Therefore, additional caution must be exercised when planning and executing treatment in the periventricular regions, which are common sites of glioma occurrence, in the face 


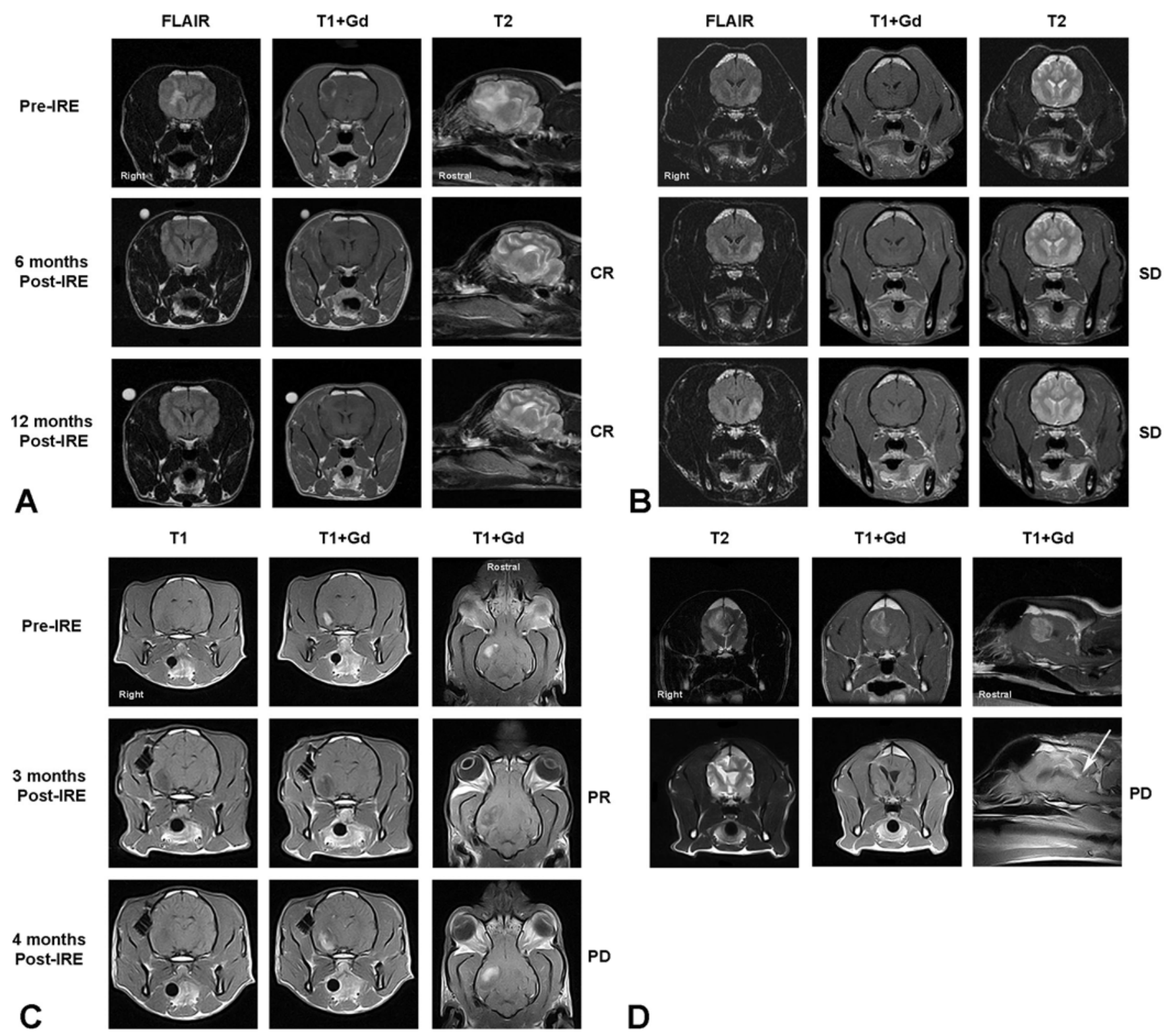

FIG. 9. Objective therapeutic responses to IRE treatment of canine intracranial gliomas determined from posttreatment MRI. A: Case 4, complete response (CR) was maintained for the 12-month study period. B: Case 6, nonenhancing (nontarget) low-grade oligodendroglioma and SD over the study period. C: Case 3, partial response (PR) 3 months after treatment with subsequent local PD at 4 months. The implanted PGP is visible on the posttreatment transverse images. D: Case 5, PD 3 months after treatment characterized by a significant reduction of contrast-enhancing tumor at the original treatment site but new distant contrast-enhancing lesions (arrow).

of tumor invasion into or attenuation of the ventricular system. ${ }^{49}$ Performance and incorporation of additional MRI studies, such as ventriculography, 3D constructive interference in steady state, or diffusion tensor imaging, into therapeutic plans may be necessary to prevent energy delivery to ventricles or eloquent brain regions.

Although fatal aspiration pneumonia was observed in $1 \mathrm{dog}$ (Case 2) in this study, it was not thought to be directly attributable to the IRE procedure. Aspiration pneumonia is a well-described and often severe complication after craniotomy and other neurosurgical procedures in dogs; it has been reported to occur in nearly $25 \%$ of dogs that undergo surgical treatment of an intracranial mass le- sion, with mortality rates that range from $23 \%$ to $58 \% .^{15,25}$ Perioperative vomiting is a known risk factor associated with aspiration in dogs with neurological disease and was present in both dogs that experienced aspiration in this study. ${ }^{15,25}$ Every dog in this study received antiemetics and gastric protectants according to the institutional standards of care for dogs that undergo craniotomy.

The majority of adverse effects observed in the 14-day acute safety window were mild to moderate (Grades 1 to 2 ) and thus were self-limiting or resolved with minor medical interventions. The most common acute adverse event in this study was Grade 1 or 2 seizures in the postoperative period, which occurred in 5 of the 7 dogs. In dogs, seizures 
are the most common clinical sign associated with brain tumors, occurring in $73 \%$ of dogs with supratentorial tumors, and all dogs in this study had secondary structural

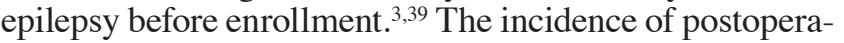
tive seizures in dogs with a brain tumor or potential influences of tumor type or specific anticonvulsant therapies on seizure outcome are unknown. Improvement in seizure control was ultimately achieved in 5 of the 6 dogs that survived to discharge.

Dog 1 was treated with IRE and adjunctive radiation therapy and was ultimately killed because of progressive encephalopathic complications. ${ }^{16}$ Postmortem examination revealed extensive regions of coagulative brain necrosis, vascular telangiectasias, and dystrophic calcification that extended from the original tumor site. These changes were interpreted as consistent with treatment-related necrosis of the brain and show that treatment-related necrosis is a potential and clinically significant complication of multimodal IRE therapy. ${ }^{16}$

\section{Procedural and Study Limitations}

We acknowledge several technical limitations that must be addressed to enable practical implementation of IRE in the clinic. There are limited data on the effects of the pulsed electric fields in heterogeneous brain tumor tissue environments. However, as more patients are treated, our computational models can be iteratively adapted to include tumor-to-tumor variability in terms of the lethal threshold required for achieving successful treatment. Currently, the generation of imaging-based computational models for treatment planning is time-consuming and requires the use of multiple software platforms. Therefore, the development of user-friendly planning systems that operate in semiautomated to fully automated ways and can be imported or integrated into the NanoKnife system is imperative. The dynamic surgical environment or intraoperative complications may introduce variations that were not accounted for in computational models, such as occurred in Case 5. Therefore, coupling the imaging-based models to real-time imaging (e.g., electrode location) or electrical (e.g., tumor properties) feedback could help further improve the planning aspects of IRE therapy and allow for some intraoperative flexibility to accommodate unexpected changes of surgical complications that might occur between the development of the original treatment plan and the confirmation of delivery of pulses with therapeutic intent. Techniques such as MR electrical impedance tomography (MREIT) have been shown to enable realtime monitoring of the electrical field distribution during electroporation-based treatments. ${ }^{26}$ We were unable to use MREIT in this study because of device and electrode probe MRI incompatibility issues.

The design of this study also did not allow for systematic investigation of the development or evolution of subacute or chronic cytoarchitectural or functional effects that IRE may have on peritumoral brain tissue. This and previous studies in rodent and canine brains have found that IRE ablations can result in acute perilesional inflammation, edema, and gliosis. ${ }^{13,21,38}$ Future research is needed to characterize the long-term effects of IRE on the brain, because any resulting changes could cause or contribute to clinically significant phenomena such as tumoral epileptogenicity. Similar to surgery, tumor ablation with the NanoKnife is planned around targeting the tumor burden as determined macroscopically via MRI and as defined by the neurosurgeon and thus will not sufficiently address microscopic tumor infiltrates extending beyond the grossly visible tumor that define malignant gliomas. ${ }^{47}$ In this study, site-distant progression occurred in 2 of the 5 dogs with a high-grade glioma, and at necropsy, 1 dog had microscopic tumor infiltrates that extended outside of the MR-targeted treatment region. Although reported cases of metastatic seeding of gliomas along surgical approach routes are rare in humans, we cannot completely exclude the possibility of iatrogenic tumor seeding in Case $5 .{ }^{42}$ However, site-distant progression in this case was observed in the deep subcortical white matter in locations caudal and remote from the sites of electrode insertions and ventricular compromise. Monitoring for disease progression along electrode trajectories is indicated in patients treated with the NanoKnife, as recommended for all cannula- or probe-based diagnostic or therapeutic interventions in cases of malignant glioma. In this study, we attempted to mitigate tumor seeding by treating from the deep to the superficial aspects of the tumors requiring treatment at multiple electrode depths along the same trajectory, thus killing tumor cells in contact with the electrode probes.

In addition to the small sample size, this study was limited by the inclusion of a cohort of dogs that were heterogeneous with respect to tumor type, tumor volume, and pretreatment KPS score. For ethical and practical reasons, the study was designed to accept all eligible dogs with supratentorial glioma. The recruitment of dogs with glioma with a priori knowledge of tumor phenotypes is challenging, because canine glioma subtypes and grades cannot be reliably distinguished by using standard diagnostic MRI sequences, ${ }^{49}$ and both antemortem diagnosticintent biopsy and surgical resection are rarely performed in client-owned dogs with glioma, as illustrated by the fact that only 1 of the 7 dogs reported here had a histopathologically confirmed glioma before study enrollment. Thus, we felt obligated to provide therapy to all the dogs with glioma whose owners consented to tumor biopsy. We also believed that the minimally invasive and precise nature of the NanoKnife IRE ablation procedure demonstrated in other organs s, $^{5,29,31}$ may have clinical utility in treating lowand high-grade gliomas for which resection is preferred, but is not feasible or practical, or for being potentially useful in certain canine or human patients who may be poor candidates for surgery or stereotactic radiosurgical treatment because of tumor location or size.

Although there are many features of dogs with spontaneous gliomas that make them attractive for preclinical studies, there are inherent limitations of the model. Currently, there is no established standard-of-care therapy for dogs with spontaneous glioma, and as was apparent in this study, many dogs entered into clinical trials receive no treatment other than what is being offered by the trial. ${ }^{6}$ Although this facilitates early case recruitment into investigative clinical trials and improves the likelihood of detecting beneficial effects of an intervention with minimal confounding variables, it may limit or otherwise alter the 
mechanisms of acquired tumor chemoresistance and radioresistance that make human gliomas notoriously difficult to treat. $^{6,48}$ The consistency and end points of preclinical studies using client-owned dogs with spontaneous brain tumors depend heavily on the owner's consent, compliance, ethics, and emotions. ${ }^{6,23}$ In addition, although numerous shared molecular and genetic aspects of tumorigenesis have been identified in canine and human gliomas, current evidence also indicates that the genetic signatures of glioma are not completely analogous between the 2 species. For example, fundamental genetic aberrations identified in human gliomas, including isocitrate dehydrogenase-1 (IDH-1) and IDH-2 mutations, which occur in $50 \%-85 \%$ of low-grade human gliomas, and 1p/19q chromosomal deletions in oligodendrogliomas, have not been identified in the dog. ${ }^{45,48}$

\section{Conclusions}

The NanoKnife system is a novel, rapid, and minimally invasive method for the ablation of spontaneous canine telencephalic gliomas with IRE. The therapeutic planning techniques used in this study should be incorporated into future iterations of the NanoKnife system to facilitate the execution of intracranial IRE procedures. In addition to the treatment of glioma for patients in which local tumor control is desired but conventional surgical resection is deemed impractical or impossible, the ablative capabilities offered by IRE may provide an alternative treatment for other brain disorders, especially those in which the intended effect is controlled destruction of a well-defined anatomical target such as benign tumors, seizure foci, or movement disorders (i.e., essential tremor).

\section{Acknowledgments}

We thank the Boo Radley Foundation for client relations and client support, Drs. Peter Brofman, Maria Killos, Jason King, Carolina Ricco, Kristin Terrio, and Deena Tiches for assistance with dog management, Drs. Christopher Arena and Michael Sano for assistance with NanoKnife treatments and planning, and Barbara Wheeler and Jennifer Rudd for technical assistance with histopathology and immunohistochemistry. We recognize the contributions made by Thomas L. Ellis, MD, to study design, data acquisition, and analyses. Dr. Ellis died on June 30, 2012, before the completion of this article.

\section{References}

1. Agerholm-Larsen B, Iversen HK, Ibsen P, Moller JM, Mahmood F, Jensen KS, et al: Preclinical validation of electrochemotherapy as an effective treatment for brain tumors. Cancer Res 71:3753-3762, 2011

2. Al-Sakere B, André F, Bernat C, Connault E, Opolon P, Davalos RV, et al: Tumor ablation with irreversible electroporation. PLoS ONE 2:e1135, 2007

3. Bagley RS, Gavin PR, Moore MP, Silver GM, Harrington ML, Connors RL: Clinical signs associated with brain tumors in dogs: 97 cases (1992-1997). J Am Vet Med Assoc 215:818-819, 1999

4. Beebe SJ, Sain NM, Ren W: Induction of cell death mechanisms and apoptosis by nanosecond pulsed electric fields (nsPEFs). Cells 2:136-162, 2013

5. Cannon R, Ellis S, Hayes D, Narayanan G, Martin RC II: Safety and early efficacy of irreversible electroporation for hepatic tumors in proximity to vital structures. J Surg Oncol 107:544-549, 2013
6. Chen L, Zhang Y, Yang J, Hagan JP, Li M: Vertebrate animal models of glioma: understanding the mechanisms and developing new therapies. Biochim Biophys Acta 1836:158-165, 2013

7. Cheung W, Kavnoudias H, Roberts S, Szkandera B, Kemp W, Thomson KR: Irreversible electroporation for unresectable hepatocellular carcinoma: initial experience and review of safety and outcomes. Technol Cancer Res Treat 12:233241, 2013

8. Davalos RV, Mir IL, Rubinsky B: Tissue ablation with irreversible electroporation. Ann Biomed Eng 33:223-231, 2005

9. Debinski W, Dickinson P, Rossmeisl JH, Robertson J, Gibo DM: New agents for targeting of IL-13RA2 expressed in primary human and canine brain tumors. PLoS ONE 8:e77719, 2013

10. Deodhar A, Dickfeld T, Single GW, Hamilton WC Jr, Thornton RH, Sofocleous CT, et al: Irreversible electroporation near the heart: ventricular arrhythmias can be prevented with ECG synchronization. AJR Am J Roentgenol 196:W330W335, 2011

11. Dickinson PJ, Roberts BN, Higgins RJ, Leutenegger CM, Bollen AW, Kass PH, et al: Expression of receptor tyrosine kinases VEGFR-1 (FLT-1), VEGFR-2 (KDR), EGFR-1, PDGFRalpha and c-Met in canine primary brain tumours. Vet Comp Oncol 4:132-140, 2006

12. Edd JF, Davalos RV: Mathematical modeling of irreversible electroporation for treatment planning. Technol Cancer Res Treat 6:275-286, 2007

13. Ellis TL, Garcia PA, Rossmeisl JH Jr, Henao-Guerrero N, Robertson J, Davalos RV: Nonthermal irreversible electroporation for intracranial surgical applications. Laboratory investigation. J Neurosurg 114:681-688, 2011

14. Engel J, Van Ness PC, Rasmussen TB, Ojemann LM: Outcome with respect to epileptic seizures, in Engels JJ (ed): Surgical Treatment of the Epilepsies, ed 2. New York: Raven Press, 1993, pp 609-621

15. Fransson BA, Bagley RS, Gay JM, Silver GM, Gokhale S, Sanders S, et al: Pneumonia after intracranial surgery in dogs. Vet Surg 30:432-439, 2001

16. Garcia PA, Pancotto T, Rossmeisl JH Jr, Henao-Guerrero N, Gustafson NR, Daniel GB, et al: Non-thermal irreversible electroporation (N-TIRE) and adjuvant fractionated radiotherapeutic multimodal therapy for intracranial malignant glioma in a canine patient. Technol Cancer Res Treat 10:73-83, 2011

17. Garcia PA, Rossmeisl JH Jr, Ellis TL, Davalos RV: Nonthermal irreversible electroporation as a focal ablation treatment for brain cancer, in: Hayat MA (ed): Tumors of the Central Nervous System. Dordrecht: Springer, 2014, Vol 12, pp 171-182

18. Garcia PA, Rossmeisl JH Jr, Neal RE II, Ellis TL, Olson JD, Henao-Guerrero N, et al: Intracranial nonthermal irreversible electroporation: in vivo analysis. J Membr Biol 236:127136,2010

19. Garcia PA, Rossmeisl JH Jr, Robertson JL, Olson JD, Johnson AJ, Ellis TL, et al: 7.0-T magnetic resonance imaging characterization of acute blood-brain-barrier disruption achieved with intracranial irreversible electroporation. PLoS ONE 7:e50482, 2012

20. Heidner GL, Kornegay JN, Page RL, Dodge RK, Thrall DE: Analysis of survival in a retrospective study of $86 \mathrm{dogs}$ with brain tumors. J Vet Intern Med 5:219-226, 1991

21. Hjouj M, Last D, Guez D, Daniels D, Sharabi S, Lavee J, et al: MRI study on reversible and irreversible electroporation induced blood brain barrier disruption. PLoS ONE 7:e42817, 2012

22. Ivorra A, Al-Sakere B, Rubinsky B, Mir LM: In vivo electrical conductivity measurements during and after tumor electroporation: conductivity changes reflect the treatment outcome. Phys Med Biol 54:5949-5963, 2009 
23. Kimmelman J, Nalbantoglu J: Faithful companions: a proposal for neurooncology trials in pet dogs. Cancer Res 67:4541-4544, 2007

24. Kirson ED, Dbalý V, Tovarys F, Vymazal J, Soustiel JF, Itzhaki A, et al: Alternating electric fields arrest cell proliferation in animal tumor models and human brain tumors. Proc Natl Acad Sci U S A 104:10152-10157, 2007

25. Kogan DA, Johnson LR, Sturges BK, Jandrey KE, Pollard RE: Etiology and clinical outcome in dogs with aspiration pneumonia: 88 cases (2004-2006). J Am Vet Med Assoc 233:1748-1755, 2008

26. Kranjc M, Bajd F, Sersa I, Woo EJ, Miklavcic D: Ex vivo and in silico feasibility study of monitoring electric field distribution in tissue during electroporation based treatments. PLoS ONE 7:e45737, 2012

27. Lipsitz D, Higgins RJ, Kortz GD, Dickinson PJ, Bollen AW, Naydan DK, et al: Glioblastoma multiforme: clinical findings, magnetic resonance imaging, and pathology in five dogs. Vet Pathol 40:659-669, 2003

28. Louis DN, Ohgaki H, Wiestler OD, Cavenee WK, Burger PC, Jouvet A, et al: The 2007 WHO classification of tumours of the central nervous system. Acta Neuropathol 114:97-109, 2007

29. Martin RC II, McFarland K, Ellis S, Velanovich V: Irreversible electroporation therapy in the management of locally advanced pancreatic adenocarcinoma. J Am Coll Surg 215:361-369, 2012

30. McGrath JT: Intracranial pathology of the dog. Acta Neuropathol 1:3-4, 1962

31. Neal RE II, Garcia PA, Robertson JL, Davalos RV: Experimental characterization and numerical modeling of tissue electrical conductivity during pulsed electric fields for irreversible electroporation treatment planning. IEEE Trans Biomed Eng 59:1076-1085, 2012

32. Narayanan G, Hosein PJ, Arora G, Barbery KJ, Froud T, Livingstone AS, et al: Percutaneous irreversible electroporation for downstaging and control of unresectable pancreatic adenocarcinoma. J Vasc Interv Radiol 23:1613-1621, 2012

33. Neal RE II, Rossmeisl JH Jr, D'Alfonso V, Robertson JL, Garcia PA, Elankumaran S, et al: In vitro and numerical support for combinatorial irreversible electroporation and electrochemotherapy glioma treatment. Ann Biomed Eng 42:475-487, 2014

34. Neal RE II, Rossmeisl JH Jr, Garcia PA, Lanz OI, HenaoGuerrero N, Davalos RV: Successful treatment of a large soft tissue sarcoma with irreversible electroporation. J Clin Oncol 29:e372-e377, 2011

35. Onik G, Mikus P, Rubinsky B: Irreversible electroporation: implications for prostate ablation. Technol Cancer Res Treat 6:295-300, 2007

36. Rossmeisl JH, Duncan RB, Huckle WR, Troy GC: Expression of vascular endothelial growth factor in tumors and plasma from dogs with primary intracranial neoplasms. Am J Vet Res 68:1239-1245, 2007

37. Rossmeisl JH Jr, Garcia PA, Daniel GB, Bourland JD, Debinski W, Dervisis N, et al: Invited review-neuroimaging response assessment criteria for brain tumors in veterinary patients. Vet Radiol Ultrasound 55:115-132, 2014

38. Rossmeisl JH Jr, Garcia PA, Roberston JL, Ellis TL, Davalos $\mathrm{RV}$ : Pathology of non-thermal irreversible electroporation (N-TIRE)-induced ablation of the canine brain. J Vet Sci 14:433-440, 2013

39. Rossmeisl JH Jr, Jones JC, Zimmerman KL, Robertson JL: Survival time following hospital discharge in dogs with palliatively treated primary brain tumors. J Am Vet Med Assoc 242:193-198, 2013

40. Salford LG, Persson BRR, Brun A, Ceberg CP, Kongstad PC, Mir LM: A new brain tumour therapy combining bleomycin with in vivo electropermeabilization. Biochem Biophys Res Commun 194:938-943, 1993
41. Sel D, Cukjati D, Batiuskaite D, Slivnik T, Mir LM, Miklavcic D: Sequential finite element model of tissue electropermeabilization. IEEE Trans Biomed Eng 52:816-827, 2005

42. Steinmetz MP, Barnett GH, Kim BS, Chidel MA, Suh JH: Metastatic seeding of the stereotactic biopsy tract in glioblastoma multiforme: case report and review of the literature. $\mathbf{J}$ Neurooncol 55:167-171, 2001

43. Stoica G, Kim HT, Hall DG, Coates JR: Morphology, immunohistochemistry, and genetic alterations in dog astrocytomas. Vet Pathol 41:10-19, 2004

44. Stoica G, Lungu G, Martini-Stoica H, Waghela S, Levine J, Smith R III: Identification of cancer stem cells in dog glioblastoma. Vet Pathol 46:391-406, 2009

45. Thomas R, Duke SE, Wang HJ, Breen TE, Higgins RJ, Linder KE, et al: 'Putting our heads together': insights into genomic conservation between human and canine intracranial tumors. J Neurooncol 94:333-349, 2009

46. Thomson KR, Cheung W, Ellis SJ, Federman D, Kavnoudias $\mathrm{H}$, Loader-Oliver D, et al: Investigation of the safety of irreversible electroporation in humans. J Vasc Interv Radiol 22:611-621, 2011

47. Valladao ML, Scarpelli KC, Metze K: Clinical utility of a life quality score in dogs with canine transmissible venereal tumor treated by vincristine chemotherapy. Arq Bra Med Vet Zootec 62:1086-1093, 2010

48. Wang Y, Jiang T: Understanding high grade glioma: molecular mechanism, therapy and comprehensive management. Cancer Lett 331:139-146, 2013

49. Young BD, Levine JM, Porter BF, Chen-Allen AV, Rossmeisl $\mathrm{JH}$, Platt SR, et al: Magnetic resonance imaging features of intracranial astrocytomas and oligodendrogliomas in dogs. Vet Radiol Ultrasound 52:132-141, 2011

50. Zinn PO, Colen RR, Kasper EM, Burkhardt JK: Extent of resection and radiotherapy in GBM: A 1973 to 2007 surveillance, epidemiology and end results analysis of 21,783 patients. Int J Oncol 42:929-934, 2013

\section{Author Contributions}

Conception and design: Rossmeisl, Garcia, Ellis, Davalos. Acquisition of data: Rossmeisl, Garcia, Pancotto, Robertson, Neal, Ellis, Davalos. Analysis and interpretation of data: Rossmeisl, Garcia, Pancotto, Robertson, Neal, Ellis, Davalos. Drafting the article: Rossmeisl, Garcia. Critically revising the article: Rossmeisl, Garcia, Pancotto, Robertson, Henao-Guerrero, Neal, Davalos. Reviewed submitted version of manuscript: Rossmeisl, Garcia, Pancotto, Robertson, Henao-Guerrero, Neal, Davalos. Approved the final version of the manuscript on behalf of all authors: Rossmeisl. Administrative/technical/material support: Rossmeisl, Garcia, Davalos. Study supervision: Rossmeisl. Animal care: Henao-Guerrero.

\section{Supplemental Information \\ Previous Presentation}

Portions of this work were presented in abstract form and in proceedings at the 2012 Gordon Research Conference held in Lucca, Italy, on July 1, 2012; the BioEM Meeting held in Thessaloniki, Greece, on June, 10, 2013; and the American College of Veterinary Internal Medicine Annual Forum held in Seattle, Washington, on June 12, 2013.

\section{Correspondence}

John H. Rossmeisl Jr., Department of Small Animal Clinical Sciences, Virginia-Maryland Regional College of Veterinary Medicine, Virginia Tech, 225 Duckpond Dr., Mail Code 0442, Blacksburg, VA 24061. email: jrossmei@vt.edu. 